\title{
Optical analysis of the poor clusters Abell 610, Abell 725, and Abell 796, containing diffuse radio sources ${ }^{\star}$
}

\author{
W. Boschin ${ }^{1,2}$, R. Barrena ${ }^{3}$, M. Girardi²,4 and M. Spolaor ${ }^{2,5}$ \\ ${ }^{1}$ Fundación Galileo Galilei - INAF, Rambla José Ana Fernández Perez 7, 38712 Breña Baja (San Antonio), Canary Islands, Spain \\ e-mail: boschin@tng.iac.es \\ 2 Dipartimento di Astronomia, Università degli Studi di Trieste, via Tiepolo 11, 34143 Trieste, Italy \\ 3 Instituto de Astrofisica de Canarias, C/Via Lactea s/n, 38205 La Laguna, Tenerife, Canary Islands, Spain \\ 4 INAF - Osservatorio Astronomico di Trieste, via Tiepolo 11, 34143 Trieste, Italy \\ 5 Centre for Astrophysics \& Supercomputing, Swinburne University, Hawthorn, VIC 3122, Australia
}

Received 20 February 2008 / Accepted 14 April 2008

\begin{abstract}
Aims. We study the dynamical status of the poor, low X-ray luminous galaxy clusters Abell 610, Abell 725, and Abell 796 (at $z=0.1$, 0.09 , and 0.16 , respectively), containing diffuse radio sources (relic, relic, and possible halo, respectively).

Methods. Our analysis is based on new spectroscopic data obtained at the William Herschel Telescope for 158 galaxies, new photometry obtained at the Isaac Newton Telescope with the addition of data recovered from the Data Release 5 of the Sloan Digital Sky Survey. We use statistical tools to select 57, 36, and 26 cluster members and to analyze the kinematics of cluster galaxies, as well as to study the $2 \mathrm{D}$ cluster structure.

Results. The low values we compute for the global line-of-sight velocity dispersion of galaxies $\left(\sigma_{\mathrm{V}}=420-700 \mathrm{~km} \mathrm{~s}^{-1}\right) \mathrm{confirm}$ that these clusters are low-mass clusters. Abell 610 shows a lot of evidence of substructure. It seems to be formed by two structures separated by $\sim 700 \mathrm{~km} \mathrm{~s}^{-1}$ in the cluster rest-frame, having comparable $\sigma_{\mathrm{V}} \sim 200 \mathrm{~km} \mathrm{~s}^{-1}$ and likely causing a velocity gradient. The velocity of the brightest cluster member (BCMI; a bright radio source) is very close to the mean velocity of the higher velocity structure. A third small, low-velocity group hosts the second brightest cluster member (BCMII). The analysis of the 2D galaxy distribution shows a bimodal distribution in the core elongated in the SE-NW direction and likely associated to BCMI and BCMII groups. Abell 725 and Abell 796, which are less sampled, show marginal evidence of substructure in the velocity space. They are elongated in the 2D galaxy distribution. For both Abell 610 and Abell 725 we shortly discuss the possible connection with the hosted diffuse radio relic.

Conclusions. Our results show that relic radio sources are likely connected with merger events, but are not limited to massive clusters. About the possible halo source in Abell 796, there is some evidence of a merger event in this non-massive cluster, but a pointed radio observation is necessary to confirm this halo.
\end{abstract}

Key words. galaxies: clusters: general - galaxies: clusters: individual: Abell 610 - galaxies: clusters: individual: Abell 725 galaxies: clusters: individual: Abell 796 - galaxies: distances and redshifts - cosmology: observations

\section{Introduction}

Clusters of galaxies are not recognized as simple relaxed structures, but rather as evolving via merging processes in a hierarchical fashion from poor groups to rich clusters. A recent aspect of these investigations is the possible connection of cluster mergers with the presence of extended, diffuse radio sources: halos and relics. These radio sources are large (up to $\sim 1 h_{70}^{-1} \mathrm{Mpc}$ ), amorphous cluster sources of uncertain origin and generally steep radio spectra (Hanisch 1982; see also Giovannini \& Feretti 2002, for a more recent review). They are rare sources that appear to be associated with very rich clusters that have undergone recent mergers. Therefore, it has been suggested by various authors that cluster halos/relics are related to recent merger activity (e.g., Tribble 1993; Burns et al. 1994; Feretti 1999).

The synchrotron radio emission of halos and relics demonstrates the existence of large scale cluster magnetic fields, of the order of $0.1-1 \mu \mathrm{G}$, and of widespread relativistic particles of energy density $10^{-14}-10^{-13} \mathrm{erg} \mathrm{cm}^{-3}$. The difficulty in explaining

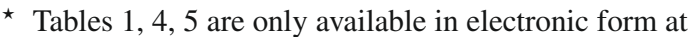
http://www. aanda.org radio halos/relics arises from the combination of their large size and the short synchrotron lifetime of relativistic electrons. The expected diffusion velocity of the electron population is of the order of the Alfven speed $\left(\sim 100 \mathrm{~km} \mathrm{~s}^{-1}\right)$, making it difficult for the electrons to diffuse over a megaparsec-scale region within their radiative lifetime. Therefore, one needs a mechanism by which the relativistic electron population can be transported over large distances in a short time, or a mechanism by which the local electron population is reaccelerated and the local magnetic fields are amplified over an extended region. The clustercluster merger can potentially supply both mechanisms (e.g., Giovannini et al. 1993; Burns et al. 1994; Röttgering et al. 1994; see also Feretti et al. 2002; Sarazin 2002, for reviews). However, the question is still debated since the diffuse radio sources are quite uncommon and only recently have we been able to study these phenomena on the basis of sufficient statistics (few dozen clusters up to $z \sim 0.3$, e.g., Giovannini et al. 1999; see also Giovannini \& Feretti 2002; Feretti 2005).

Growing evidence of the connection between diffuse radio emission and cluster merging is based on X-ray data (e.g., Böhringer \& Schuecker 2002; Buote 2002). Studies based on 
a large number of clusters have found a significant relation between the radio and the X-ray surface brightness (Govoni et al. 2001a,b) and between the presence of radio halos/relics and irregular and bimodal X-ray surface brightness distribution (Schuecker et al. 2001).

Optical data are a powerful way to investigate the presence and the dynamics of cluster mergers, too (e.g., Girardi \& Biviano 2002). The spatial and kinematical analysis of member galaxies allow us to detect and measure the amount of substructure, to identify and analyze possible pre-merging clumps or merger remnants. This optical information is complementary to X-ray information since galaxies and intracluster medium react on different timescales during a merger (see, e.g., numerical simulations by Roettiger et al. 1997). Unfortunately, to date optical data are lacking or are poorly exploited to investigate the phenomenon of diffuse radio sources. The sparse literature contains a few individual clusters (e.g., Barrena et al. 2002; Mercurio et al. 2003; Maurogordato et al. 2008).

In this context, we are conducting an intensive observational and data analysis program to study the internal dynamics of radio clusters by using member galaxies. Clusters already analyzed are: Abell 2219 (Boschin et al. 2004); Abell 2744 (Boschin et al. 2006); Abell 697 (Girardi et al. 2006); Abell 773 (Barrena et al. 2007a); Abell 115 (Barrena et al. 2007b). These are all massive clusters and, indeed, to date analyzed clusters containing a radio halo or a relic source have a large gravitational mass (larger than $0.7 \times 10^{15} h_{70}^{-1} M_{\odot}$ within $2 h_{70}^{-1} \mathrm{Mpc}$; see Giovannini \& Feretti 2002). From the theoretical point of view, a large mass is an expected property in radio clusters since the energy available to accelerate relativistic particles in a merger scales as $\sim M^{2}$, as discussed by Buote (2001).

In this paper, we report our results about three poor Abell clusters: Abell 610, Abell 725, and Abell 796 (hereafter A610, A725, and A796) having Abell richness (i.e., the number of galaxies within 1 Abell radius and magnitude between $m_{3}$ and $m_{3}+2$; see Abell et al. 1989) of 46, 36, and 56 galaxies respectively. These clusters contain a radio relic or a (possible) radio halo. In particular, A610 exhibits a relic source confirmed by pointed observations (Giovannini \& Feretti 2000); A725 presents a relic source well visible in survey data (Kempner \& Sarazin 2001); and A796 possibly hosts a halo source visible in survey data (Kempner \& Sarazin 2001) which needs to be confirmed. To date, no measure of internal velocity dispersion and/or X-ray temperature have been reported in the literature. We have recently carried out spectroscopic observations with the William Herschel Telescope giving new redshift data for 158 galaxies in the field of these clusters, as well as photometric observations at the Isaac Newton Telescope for A725. We recover additional photometric and spectroscopic information from the Data Release 5 of the Sloan Digital Sky Survey (SDSS DR5).

The paper is organized as follows. We present the new optical data in Sect. 2. We present the relevant analyses and conclusions in Sects. 3-5 for A610, A725, and A796, respectively. We summarize and discuss our results in Sect. 6.

Unless otherwise stated, we give errors at the $68 \%$ confidence level (hereafter c.l.). Throughout the paper, we assume a flat cosmology with $\Omega_{\mathrm{m}}=0.3, \Omega_{\Lambda}=0.7$ and $H_{0}=70$ $h_{70} \mathrm{~km} \mathrm{~s}^{-1} \mathrm{Mpc}^{-1}$. For this cosmological model, $1^{\prime}$ corresponds to 108,102 , and $163 h_{70}^{-1} \mathrm{kpc}$ at the A610, A725, and A796 redshift.

\section{Data sample}

\subsection{Spectroscopy}

We carried out multi-object spectroscopic observations of A610, A725, and A796 in November 2004 and January 2005. We used AF2/WYFFOS, the multi-object, wide-field, fiber spectrograph working at the prime focus of the $4.2 \mathrm{~m}$ William Herschel Telescope (WHT, Island of La Palma, Spain). This spectrograph is equipped with optical fibers each of 1.6 arcsec diameter. We used the grism R300B in combination with the 2-chip EEV $4 \mathrm{~K} \times 4 \mathrm{~K}$ pixel mosaic (pixel size $13.5 \mu \mathrm{m}$ ) working in binning $2 \times 2$. In the case of A610 and A725, we acquired two exposures of $1800 \mathrm{~s}$ for two fiber configurations, in the case of A796 we acquired three exposures of $1800 \mathrm{~s}$ for two fiber configurations. We performed wavelength calibration with the helium lamp. Reduction of spectroscopic data was carried out with the IRAF package ${ }^{1}$.

We determined radial velocities with the cross-correlation technique (Tonry \& Davis 1979) implemented in the RVSAO package (developed at the Smithsonian Astrophysical Observatory Telescope Data Center). Each spectrum was correlated against six templates for a variety of galaxy spectral types: E, S0, Sa, Sb, Sc and Ir (Kennicutt 1992). The template producing the highest value of $\mathcal{R}$, i.e., the parameter given by RVSAO and related to the signal-to-noise of the correlation peak, was chosen. Moreover, all the spectra and their best correlation functions were examined visually to verify the redshift determination. In one case (galaxy ID 127 of A610, see Table 1), we took the EMSAO redshift as a reliable estimate of the redshift. We obtained redshifts for 62, 51, 45 galaxies for A610, A725, A796.

For ten galaxies we obtained two redshift determinations of similar quality. This allows us to obtain a more rigorous estimate for the redshift errors since the nominal errors as given by the cross-correlation are known to be smaller than the true errors (e.g., Malumuth et al. 1992; Bardelli et al. 1994; Ellingson \& Yee 1994; Quintana et al. 2000). For these ten galaxies we fit the first determination vs. the second one by using a straight line and considering errors in both coordinates (e.g., Press et al. 1992). The fitted line agrees with the one-to-one relation, but, when using the nominal cross-correlation errors, the small value of the $\chi^{2}$ probability indicates a poor fit, suggesting the errors are underestimated. Only when nominal errors are multiplied by a factor of $\sim 1.3$ can the observed scatter be justified. We therefore assume hereafter that true errors are larger than nominal cross-correlation errors by a factor 1.3. For the ten galaxies we used the weighted mean of the two redshift determinations and the corresponding error.

For A610 and A796 we add galaxies with spectroscopic and/or photometric data found in the SDSS DR5. For A610, we find 147 galaxies within a radius of $30^{\prime}$ from the cluster center given by Abell et al. (1989). This radius is about the double of that sampled by our data and well larger than the virial radius of the cluster (see Sect. 3.1), and thus will be useful to study the cluster periphery, too. Out of 147 SDSS galaxies, 44 are in common with our WHT galaxies. We fit SDSS redshift determination vs. our determination finding that the fitted line well agrees with the one-to-one relation. Again, the small value of the $\chi^{2}$ probability indicates a poor fit, suggesting the errors are

\footnotetext{
1 IRAF is distributed by the National Optical Astronomy Observatories, which are operated by the Association of Universities for Research in Astronomy, Inc., under cooperative agreement with the National Science Foundation.
} 
underestimated, but we prefer to not apply any correction since the measurements come from two different sources. We combine WHT and SDSS data using the weighted mean of the two redshift determinations and the corresponding error. Our final spectroscopic catalog consists of 165 galaxies. As for A796, we find 72 SDSS galaxies - out of which there are 18 in common with our WHT galaxies - and compile a final spectroscopic catalog of 99 galaxies.

\subsection{Photometry}

Our photometric observations were carried out with the Wide Field Camera (WFC), mounted at the prime focus of the $2.5 \mathrm{~m}$ Isaac Newton Telescope (INT, Island of La Palma, Spain). We observed A725 in December 2004 in photometric conditions with a seeing of about $1.8^{\prime \prime}$ in $R_{\mathrm{H}}$ (Harris) band and $2.5^{\prime \prime}$ in $B_{\mathrm{H}}$.

The WFC consists of a four-CCD mosaic covering a $33^{\prime} \times 33^{\prime}$ field of view, with only a $20 \%$ marginally vignetted area. We took 9 exposures of $720 \mathrm{~s}$ in $B_{\mathrm{H}}$ and another 13 exposures of $360 \mathrm{~s}$ in $R_{\mathrm{H}}$ filters (a total of $6480 \mathrm{~s}$ and $4680 \mathrm{~s}$ in the two bands, respectively). We developed a dithering pattern to build a "supersky" frame that was used to correct our images for fringing patterns (Gullixson 1992). In addition, the dithering helped us to clean cosmic rays and avoid gaps between the CCDs in the final images. The complete reduction process (including flat fielding, bias subtraction, and bad-column elimination) yielded a final coadded image where the variation of the sky was lower than $1.0 \%$ within a region of $13^{\prime}$ radius from the center of the cluster.

In order to match the photometry of several filters, a good astrometric solution is needed. The astrometry has to take into account the field distortions present in the WFC full frame. Using IRAF tasks and taking as a reference the USNO B1.0 catalog, we were able to find an accurate astrometric solution $\left(\mathrm{rms} \sim 0.5^{\prime \prime}\right)$ across the full frame.

We performed the photometric calibration with Landolt standard fields observed in December 2007 using the $80 \mathrm{~cm}$ telescope at Teide Observatory. We finally identified galaxies in our $B_{\mathrm{H}}$ and $R_{\mathrm{H}}$ images and measured their magnitudes with the SExtractor package (Bertin \& Arnouts 1996) and the AUTOMAG procedure. In a few cases (e.g., objects close to very bright and saturated stars) the standard SExtractor photometric procedure failed. In these cases, by performing an isophotal analysis (task bmodel in IRAF) we eliminate the bright objects. Then, we reran SExtractor to estimate the magnitudes of faint targets close to bright stars.

We transformed all Harris magnitudes into the JohnsonCousins system (Johnson \& Morgan 1953; Cousins 1976). We used $B=B_{\mathrm{H}}+0.13$ and $R=R_{\mathrm{H}}$ as derived from the Harris filter characterization (http: //www . ast . cam. ac . uk/ wfcsur/ technical/photom/colours/) and assuming a $B-V \sim 1.0$ for E-type galaxies (Poggianti 1997). As a final step, we estimated and corrected the galactic extinction $A_{B} \sim 0.23, A_{R} \sim$ 0.14 and $E(B-V)=0.05$ from Burstein \& Heiles's (1982) reddening maps.

The photometric sample of galaxies in the field of A725 is complete down to $B=22.5$ (24.4) and $R=20.6$ (22.1) for $S / N=5$ (3) within a square region of $33^{\prime} \times 33^{\prime}$ centered on $\mathrm{RA}=09^{\mathrm{h}} 00^{\mathrm{m}} 50^{\mathrm{s}}$ and Dec $=+62^{\circ} 39^{\prime} 00^{\prime \prime}(\mathrm{J} 2000)$, and with a void of $11^{\prime} \times 11^{\prime}$ in the NW of the field. As for the spectroscopic sample, it is $\sim 25 \%$ complete down to $R=17.5$ in the whole field and $\sim 45 \%$ complete down to $R=17.5$ within $1 \mathrm{Mpc}$ from the cluster center.

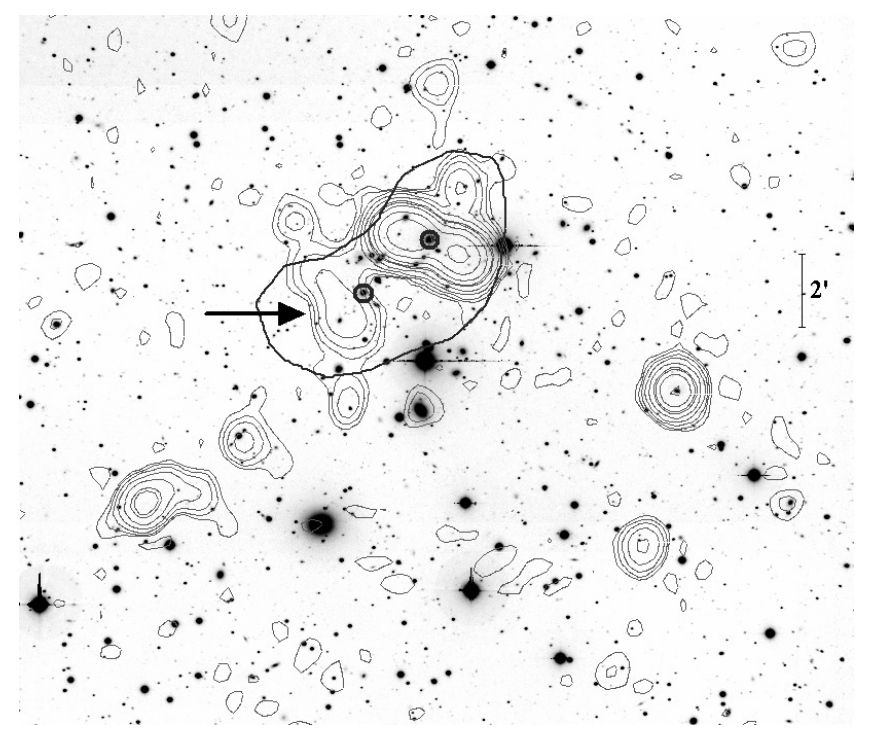

Fig. 1. $R$-band image of A610 (data taken with the WFC camera of the INT) with, superimposed, the contour levels of a VLA radio image at $20 \mathrm{~cm}$ (thin contours; Giovannini \& Feretti 2000). To avoid confusion, only one isodensity contour (thick contour) of the spatial distribution of the likely cluster members (see text) is shown. Two thick circles highlight the positions of the two brightest cluster members. The arrow shows the position of the radio relic. North is at the top and east to the left.

For A610 and A796, we use public photometric data from the SDSS DR5. In particular, we use $r^{\prime}, i^{\prime}, z^{\prime}$ magnitudes, already corrected for the Galactic extinction and consider galaxies within a radius of $30^{\prime}$ from the cluster center given by Abell et al. (1989). In the case of A610, our spectroscopic sample is $40 \%$ complete down to $r^{\prime}=17.5$ within $1 \mathrm{Mpc}$ from the cluster center. As for A796, our spectroscopic sample is $\sim 20 \%$ complete down to $r^{\prime}=19$ within $1 \mathrm{Mpc}$ from the cluster center.

\section{Abell 610}

A610 is a poor Abell cluster (Abell richness class = 0; Abell et al. 1989) located in the background of the rich nearby cluster $\mathrm{ZwCl} 0752.9+2833$ (at $z=0.015-0.027$ according to NED). Kowalski et al. (1992) reported a $2 \sigma$ upper limit for the X-ray luminosity $L_{X}(2-6 \mathrm{keV})=0.58 \times 10^{44} h_{75}^{-2} \mathrm{erg} \mathrm{s}^{-1}$ (in their cosmology).

We identified the well known bright radio source B2 0756+27 (ID 91 in our catalog, see below) as a double radio galaxy associated with the optically brightest, giant elliptical of the cluster (Valentijn 1979; see also Owen et al. 1992). For this galaxy Owen et al. (1995) measured a redshift of $z=$ $0.0991 \pm 0.0002$ and detected $\mathrm{H} \alpha+\mathrm{NII}$ emission lines. This WAT radio galaxy has been recently studied by Jetha et al. (2006), too. The presence of a diffuse radio source at Southeast from the radio galaxy (see Fig. 1) was suggested by Valentijn (1979) and then confirmed and classified as a relic by Giovannini \& Feretti (2000).

Table 1 lists our velocity catalog and Fig. 2 shows the finding chart for the 165 galaxies in the field of A610. In Table 1, for each galaxy we provide: identification number ID (Col. 1), right ascension and declination, $\alpha$ and $\delta$ (J2000, Col. 2); $r^{\prime}$ SDSS magnitudes (Col. 3); heliocentric radial velocities, $v=c z_{\odot}$ (Col. 4) with errors, $\Delta v$ (Col. 5); source of velocity data (W: WHT, S: SDSS, W+S: combined WHT and SDSS) in Col. 6. 


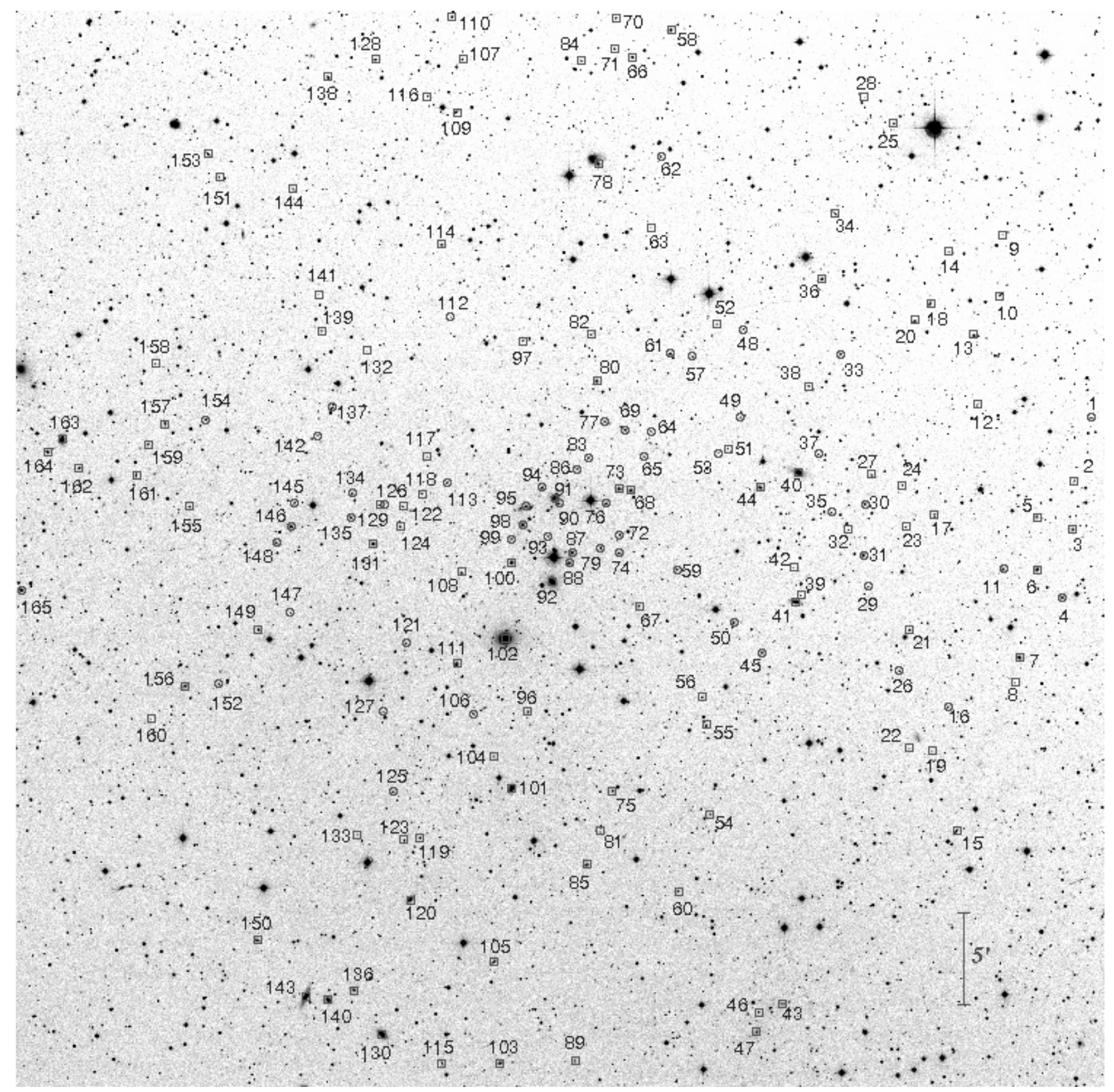

Fig. 2. DSS2 R-band image of A610 (North at the top and East to the left). Galaxies with successful velocity measurements are labeled as in Table 1 . Circles and boxes indicate cluster members and non-member galaxies, respectively.

The typical error on radial velocity as given by the median value is $38 \mathrm{~km} \mathrm{~s}^{-1}$.

\subsection{Member selection and global properties}

To select cluster members from the 165 galaxies having redshifts, we use the adaptive-kernel method (hereafter DEDICA, Pisani 1993, 1996; see also Fadda et al. 1996; Girardi et al. 1996; Girardi \& Mezzetti 2001). We find significant peaks in the velocity distribution at $>99 \%$ c.l. This procedure detects A610 as a one-peaked structure, populated by 61 galaxies with $27598 \leq v \leq 31213 \mathrm{~km} \mathrm{~s}^{-1}$ (see Fig. 3). Out of the non-member galaxies, 70 and 34 are foreground and background galaxies, respectively.
All the galaxies assigned to the A610 peak are analyzed in the second step, which uses the combination of position and velocity information. We apply the procedure of the "shifting gapper" by Fadda et al. (1996). This procedure rejects galaxies that are too far in velocity from the main body of galaxies and within a fixed bin that shifts along the distance from the cluster center. The procedure is iterated until the number of cluster members converges to a stable value. Following Fadda et al. (1996) we use a gap of $1000 \mathrm{~km} \mathrm{~s}^{-1}$ in the cluster restframe and a bin of $0.6 h_{70}^{-1} \mathrm{Mpc}$, or large enough to include 15 galaxies. As for the cluster center, we consider the position of the dominant galaxy $\left(\mathrm{RA}=07^{\mathrm{h}} 59^{\mathrm{m}} 17^{\mathrm{s}} \cdot 10\right.$, Dec $=+27^{\circ} 09^{\prime} 16^{\prime \prime} 1$ (J2000)) which was identified with the radio galaxy producing the source B2 $0756+27$ (see above). The shifting-gapper procedure rejects another four galaxies as non-members (cross 


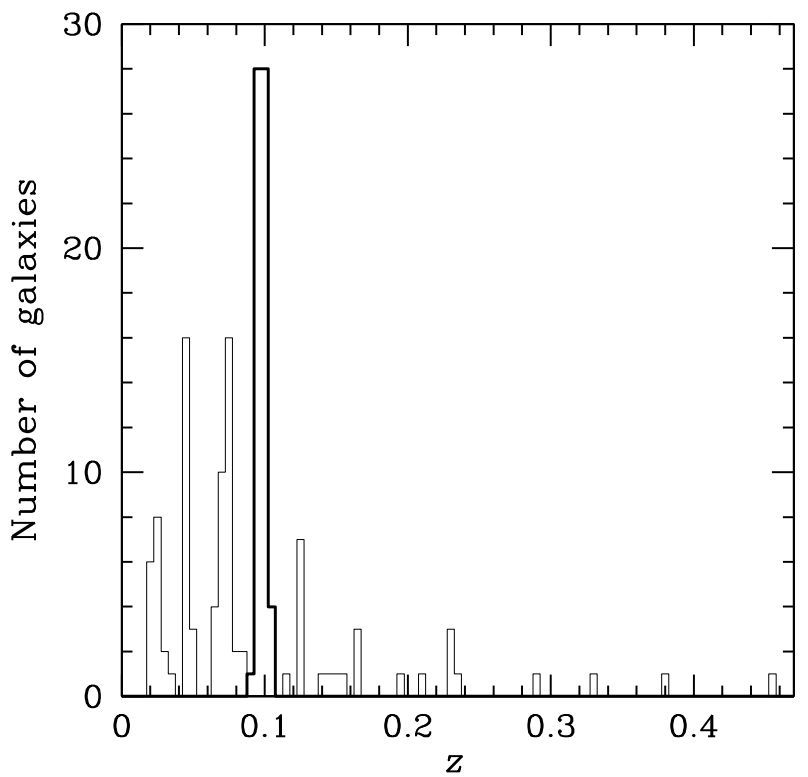

Fig. 3. A610: redshift galaxy distribution. The solid line histogram refers to galaxies assigned to the cluster according to the DEDICA reconstruction method.

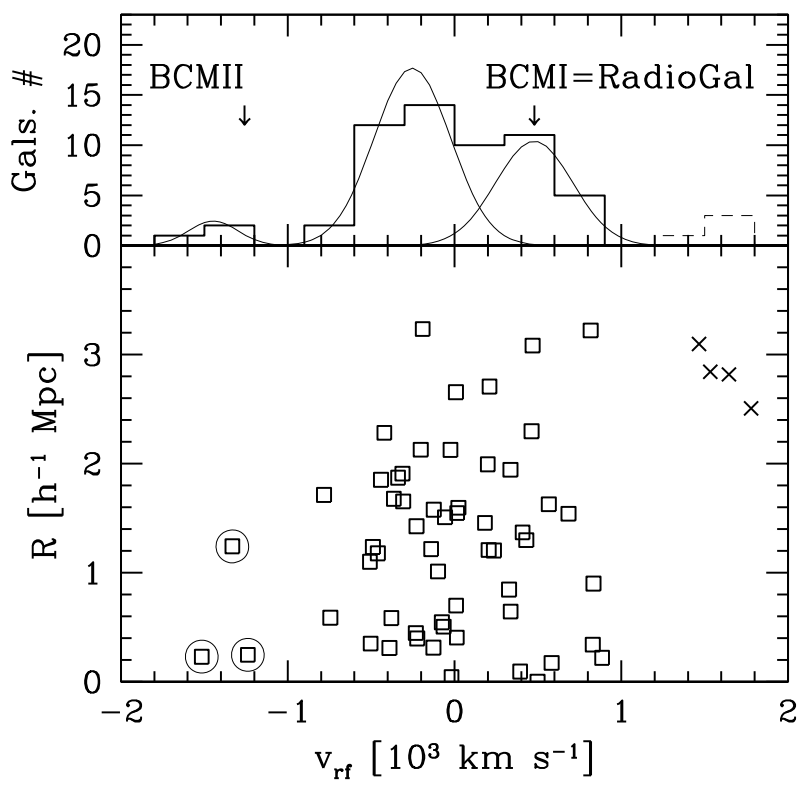

Fig. 4. A610. Bottom panel: rest-frame velocity vs. projected clustercentric distance for the 61 galaxies in the main peak (Fig. 3) showing galaxies detected as interlopers by our "shifting gapper" procedure (crosses). Squares indicate galaxies forming the sample of the 57 member galaxies. The three galaxies with the lowest velocities are rejected to build Sample2 (see text). Top panel: velocity distribution of the 57 cluster members and 4 rejected galaxies (solid and dashed histograms). Arrows correspond to the two brightest cluster members. The three Gaussians correspond to the three KMM groups (see Table 2).

symbols in Fig. 4). Thus, the member selection procedure leads to a sample of 57 cluster members (see Table 1 and Fig. 4).

By applying the biweight estimator to the cluster members (Beers et al. 1990), we compute a mean cluster redshift of $\langle z\rangle=0.0976 \pm 0.0002$, i.e., $\langle v\rangle=29255 \pm 66 \mathrm{~km} \mathrm{~s}^{-1}$. We estimate the LOS velocity dispersion, $\sigma_{\mathrm{V}}$, by using the biweight estimator and applying the cosmological correction and the standard correction for velocity errors (Danese et al. 1980). We
Table 2. A610: results of the kinematical analysis.

\begin{tabular}{lrcccc}
\hline \hline Sample & $N_{\mathrm{g}}$ & $\begin{array}{c}\langle v\rangle \\
\mathrm{km} \mathrm{s}^{-1}\end{array}$ & $\begin{array}{c}\sigma_{\mathrm{V}}^{a} \\
\mathrm{~km} \mathrm{~s}^{-1}\end{array}$ & $\begin{array}{c}R_{\mathrm{vir}} \\
\mathrm{Mpc}\end{array}$ & $\begin{array}{c}\text { Mass }\left(<R_{\mathrm{vir}}\right) \\
10^{14} M_{\odot}\end{array}$ \\
\hline Whole system & 57 & $29255 \pm 66$ & $496_{-48}^{+73}$ & 1.15 & $2.3_{-0.5}^{+0.8}$ \\
Whole system Sample2 & 54 & $29283 \pm 58$ & $426_{-39}^{+38}$ & 0.99 & $1.8_{-0.4}^{+0.4}$ \\
Virialized subsample & 22 & $29312 \pm 106$ & $480_{-59}^{+71}$ & 1.11 & $1.8_{-0.5}^{+0.6}$ \\
KMM1 & 3 & $27766 \pm 89$ & $(118)$ & - & - \\
KMM2 & 34 & $29017 \pm 40$ & $227_{-21}^{+38}$ & 0.53 & $0.22_{-0.07}^{+0.09}$ \\
KMM3 & 20 & $29767 \pm 54$ & $235_{-29}^{+32}$ & 0.54 & $0.25_{-0.07}^{+0.09}$ \\
\hline
\end{tabular}

${ }^{a}$ We use the biweigth estimator by Beers et al. (1990). For the sample with 3 galaxies we indicate the standard dispersion estimate.
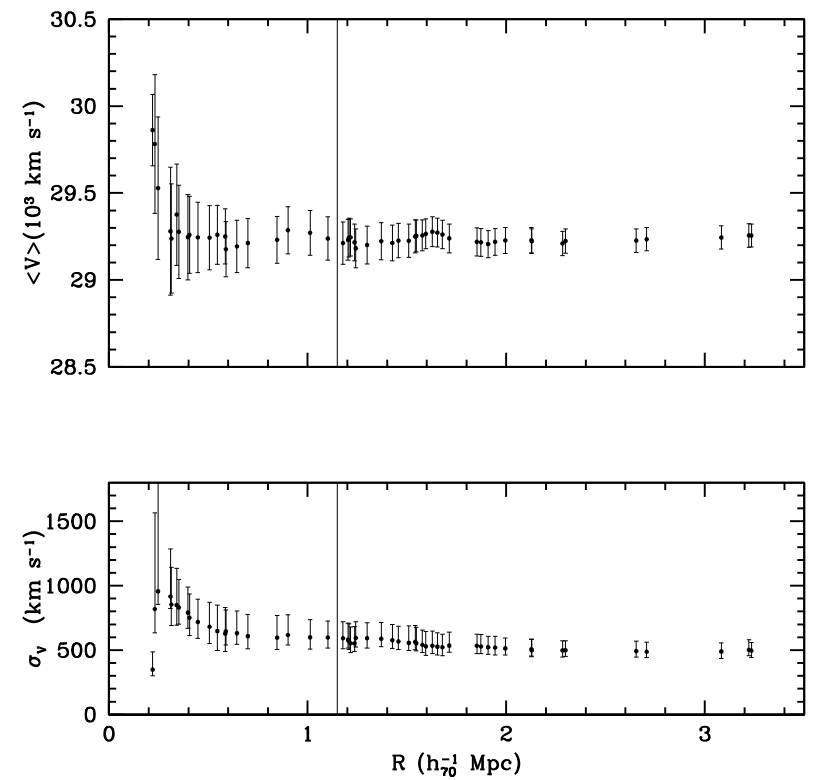

Fig. 5. A610: integral profiles of mean velocity and LOS velocitydispersion are shown in top and bottom panels, respectively. The mean and dispersion at a given (projected) radius from the cluster center is estimated by considering all galaxies within that radius - the first value computed on the five galaxies closest to the center. The error bands at the $68 \%$ c.l. are also shown. The faint vertical lines give the radius of the virialized region.

obtain $\sigma_{\mathrm{V}}=496_{-48}^{+73} \mathrm{~km} \mathrm{~s}^{-1}$, where errors are estimated through a bootstrap technique.

Here we compute the mass of A610 assuming that the system is in dynamical equilibrium. Following the prescriptions of Girardi \& Mezzetti (2001) - in particular see their Eq. (1) after introducing the scaling with $H(z)$ - we assume for the radius of the quasi-virialized region $R_{\mathrm{vir}}=0.17 \times \sigma_{\mathrm{V}} / H(z)=$ $1.15 h_{70}^{-1} \mathrm{Mpc}$ (see also Eq. (8) of Carlberg et al. 1997 for $R_{200}$ ). Therefore, our spectroscopic catalog samples the whole virialized region of the cluster.

One can compute the mass using the virial theorem (Limber \& Mathews 1960; see also Girardi et al. 1998) under the assumption that mass follows galaxy distribution: $M=3 \pi / 2 \cdot \sigma_{\mathrm{V}}^{2} R_{\mathrm{PV}} / G$ is the standard virial mass, $R_{\mathrm{PV}}$ a projected radius (equal to two times the harmonic radius). Figure 5 shows that the estimate of $\sigma_{\mathrm{V}}$ is robust when computed within a large cluster region, thus we consider the global value (see Fig. 5 of Girardi et al. 2006; and Fadda et al. 1996, for other examples). The value of $R_{\mathrm{PV}}$ depends on the size of the region considered so that the computed mass increases (but not linearly) with the increasing region 


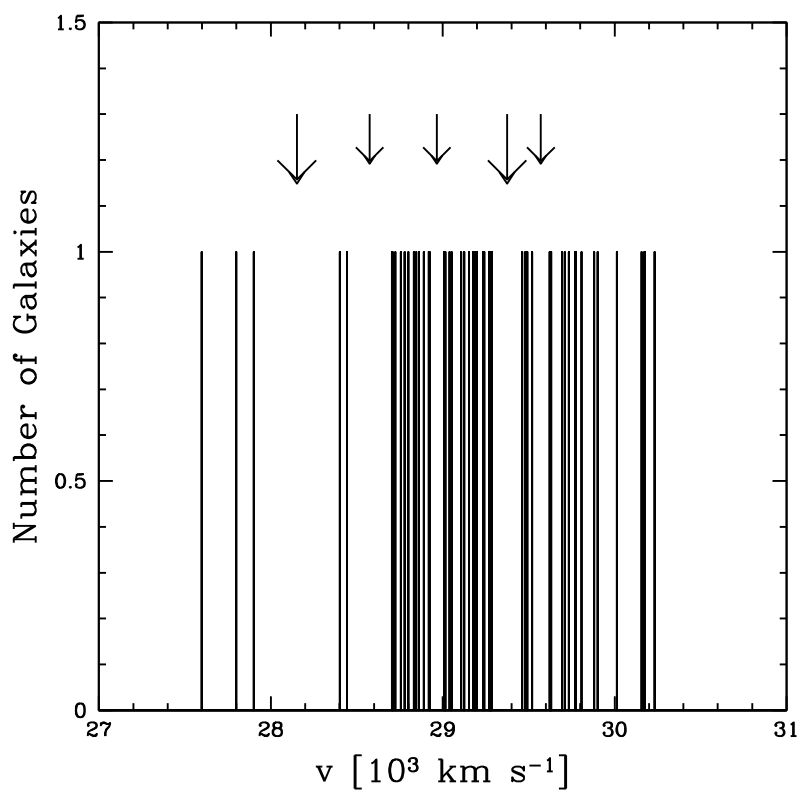

Fig. 6. A610: stripe density plot where the arrows indicate the positions of the significant gaps. The two most important gaps are indicated by the two big arrows.

considered. Considering the 24 galaxies within $R_{\text {vir }}$ we obtain $R_{\mathrm{PV}}=0.87 \pm 0.11 h_{70}^{-1} \mathrm{Mpc}$.

We obtain a virial mass $M\left(<R_{\text {vir }}=1.15 \quad h_{70}^{-1} \mathrm{Mpc}\right)=$ $2.3_{-0.6}^{+0.8} \times 10^{14} h_{70}^{-1} M_{\odot}$. Notice that in this case we do not apply the $20 \%$ surface pressure term correction (e.g., The \& White 1986; Carlberg et al. 1997; Girardi et al. 1998) since we use the value of the velocity dispersion as computed within a very large radius.

We also consider an alternative sample of 54 galaxies Sample2 - rejecting the three galaxies with lowest velocity, which are separated by a gap of $\sim 450 \mathrm{~km} \mathrm{~s}^{-1}$ in the reference frame from the whole velocity distribution of the cluster (see Fig. 4). Since this is a poor cluster such a value for a gap is quite anomalous (see also below Sect. 3.2). The properties of Sample2 are listed in Table 2.

We also present the analysis of the 22 galaxies which are the subsample of Sample2 contained within $R_{\text {vir }}$ (hereafter the "virialized subsample"), to consider galaxies likely belonging to the true internal, virialized structure.

\subsection{Analysis of the spectroscopic sample}

We analyze the velocity distribution to look for possible deviations from Gaussianity that could provide important signatures of complex dynamics. For the following tests the null hypothesis is that the velocity distribution is a single Gaussian.

We estimate three shape estimators, i.e., the kurtosis, the skewness, and the scaled tail index (see, e.g., Beers et al. 1991). The value of the skewness $(-0.530)$ shows a marginal evidence that the velocity distribution differs from a Gaussian at the 90-95\% c.l. (see Table 2 of Bird \& Beers 1993). Moreover, the W-test (Shapiro \& Wilk 1965) marginally rejects the null hypothesis of a Gaussian parent distribution at the $90 \%$ c.l. The analysis of Sample 2 also shows a marginal sign of non-Gaussianity having the kurtosis a value of 3.693 (at 90-95\% c.1.).

Then we investigate the presence of gaps in the velocity distribution. A weighted gap in the space of the ordered velocities
Table 3. A610: results of the weighted-gap analysis.

\begin{tabular}{lcllc}
\hline \hline Sample & $N_{\text {gals,prec }}$ & $\begin{array}{l}v_{\text {prec }} \\
\mathrm{km} \mathrm{s}^{-1}\end{array}$ & Size & Prob.of.detect. \\
\hline Whole sample & 3 & 27901 & 2.79 & $6.0 \mathrm{E}-3$ \\
Whole sample & 5 & 28444 & 2.55 & $1.4 \mathrm{E}-2$ \\
Whole sample & 17 & $28924^{a}$ & 2.32 & $3.0 \mathrm{E}-2$ \\
Whole sample & 36 & $29287^{a}$ & 3.56 & $5.0 \mathrm{E}-4$ \\
Whole sample & 5 & $29521^{a}$ & 2.73 & $1.4 \mathrm{E}-2$ \\
Virialized sample & 14 & $29277^{b}$ & 3.19 & $2.0 \mathrm{E}-3$ \\
\hline
\end{tabular}

${ }^{a}$ Gaps also found in Sample2.

${ }^{b}$ This gap corresponds to the most significant in the whole sample.

is defined as the difference between two contiguous velocities, weighted by the location of these velocities with respect to the middle of the data. We obtain values for these gaps relative to their average size, precisely the midmean of the weighted-gap distribution. We look for normalized gaps larger than 2.25 since in random draws of a Gaussian distribution they arise at most in about $3 \%$ of the cases, independently of the sample size (Wainer and Schacht 1978; see also Beers et al. 1991). We find five significant gaps (see Fig. 6), the main one lying in the body of the distribution at $v \sim 29500 \mathrm{~km} \mathrm{~s}^{-1}$ and the secondary one separates the three galaxies with the lowest velocity from the body of the velocity distribution. In Table 3 we list the number of galaxies and the velocity of the object preceding the gap, the normalized size (i.e., the "importance") of the gap itself, and the probability of finding a normalized gap of this size with the same position in a normal distribution (as computed with ROSTAT package, Beers et al. 1990). The importance of the main gap is confirmed by the analysis of Sample2.

We use the results of the gap analysis to determine the first guess when using the Kaye's mixture model (KMM) to find a possible group partition of the velocity distribution (as implemented by Ashman et al. 1994). The KMM algorithm fits an user-specified number of Gaussian distributions to a dataset and assesses the improvement of that fit over a single Gaussian. In addition, it provides the maximum-likelihood estimate of the unknown n-mode Gaussians and an assignment of objects into groups. KMM is most appropriate in situations where theoretical and/or empirical arguments indicate that a Gaussian model is reasonable. The Gaussian is valid in the case of cluster velocity distributions, where gravitational interactions drive the system toward a relaxed configuration with a Gaussian velocity distribution. However, one of the major uncertainties of this method is the optimal choice of the number of groups for the partition. Using the results of the gap analysis we try to fit two and three velocity groups on the basis of the two most important gaps.

We find that a two-groups partition is a significantly better descriptor of the velocity distribution with respect to a single Gaussian at the 90-92\% c.l. (homoscedastic and heteroscedastic cases) where the first group is given by the three lowest velocity galaxies. Moreover, we find a significant three-groups partition of 3-34-20 galaxies (at the $96.8 \%$ c.l. in the homoscedastic case). The KMM analysis of Sample2 confirms the presence of the two groups with 34 and 20 galaxies (at the $~ 90 \%$ c.l.). Table 2 lists the results for the kinematical analysis of the groups with 3, 34, and 20 galaxies and Fig. 4 shows the corresponding Gaussians. We also list the virial radii and masses of the groups KMM2 and KMM3. They are computed assuming the dynamical equilibrium and adopting an alternative estimate for $R_{\mathrm{PV}}$ useful when the centers of the systems are not well defined (see Barrena et al. 2007b, their Sect. 4). However, we point out that 
the uncertainty in KMM membership assignments leads to an artificial truncation of the tails of the velocity distributions, thus the velocity dispersions of the two groups are underestimated (see Bird 1994). As a consequence, the masses of KMM2 and KMM3 should be considered only approximated lower limits.

Notice that the first and the second brightest galaxies of the cluster (galaxy IDs 91 and 99; hereafter we refer to them as BCMI and BCMII) are assigned to KMM3 and KMM1 groups. In particular, BCMI has a velocity very close to the mean velocity of its host KMM3 system, while it shows evidence of peculiarity according to the Indicator test by Gebhardt \& Beers (1991, at the $>95 \%$ c.l.) with respect to the whole system. As for $\mathrm{KMM} 2$, it does not host very luminous galaxies. Indeed, not all galaxy systems are characterized by particularly bright galaxies. In particular, dominant galaxies are often absent in poorly evolved structures (see, for instance, the "irregular" and "flat" clusters according to the definition of Struble \& Rood 1982, 1984). The existence of a correlation between positions and velocities of cluster galaxies is a footprint of real substructures. To investigate the velocity field of the A610 complex we divide galaxies in a low and a high velocity samples by using the median cluster velocity and check the difference between the two distributions of galaxy positions. The two distributions are different at the $92.3 \%$ c.l. according to the 2DKS-test. The same analysis on Sample2 leads to a difference at the $98.4 \%$ c.1. In order to estimate the direction of the velocity gradient we perform a multiple linear regression fit to the observed velocities with respect to the galaxy positions in the plane of the sky (see also den Hartog \& Katgert 1996; Girardi et al. 1996). We find a position angle on the celestial sphere of PA $=184_{-19}^{+25}$ degree (measured counter-clockwise from north, i.e., higher-velocity galaxies lie in the southern region), but marginally significant (at the $90 \%$ c.l.).

Looking for a correlation between positions and velocities, we also compare the KMM groups two-by-two by applying the 2DKS-test to the galaxy position of member galaxies. When comparing KMM2 with KMM3 we obtain a difference at the $99.88 \%$ c.l. and a marginal difference when comparing KMM1 with KMM2 (at the $90 \%$ c.1.). Figure 7 shows as the higher velocity KMM3 group lies SW with respect KMM2 group.

When limiting our analysis to the "virialized subsample" we find again: a marginal evidence of non-Gaussianity (at the $90-95 \%$ c.l. from the kurtosis of -0.849); the gap at $\sim 29500 \mathrm{~km} \mathrm{~s}^{-1}$; a different spatial distribution between low and high velocity populations (at the $\gtrsim 95 \%$ c.l.). The presence of a velocity gradient is now significant at the $94 \%$ c.l. with a value of $\mathrm{PA}=64_{-42}^{+59}$ and, in fact, the high velocity KMM3 galaxies lie $\mathrm{NE}$ in the central cluster region (see Fig. 7).

\section{3. $2 D$ galaxy distribution}

When applying the DEDICA method to the 2D distribution of the 57 cluster members we find two very significant peaks (at the $>99.9$ c.l., see Fig. 8). The position of the highest peak is close to the location of the brightest cluster member. The secondary peak lies at $\sim 15^{\prime}$ East $\left(\sim 1.6 h_{70}^{-1} \mathrm{Mpc}\right)$ with respect to the BCMI. We compare the mean velocity of galaxies belonging to the main peak of our 2D analysis with that of galaxies belonging to the eastern secondary peak. We find no difference. This explains why the eastern structure is not detected in the analyses of Sect. 3.2.

Our spectroscopic data suffer from magnitude incompleteness. To overcome this limit we recover the photometric catalog extracted from the SDSS DR5.

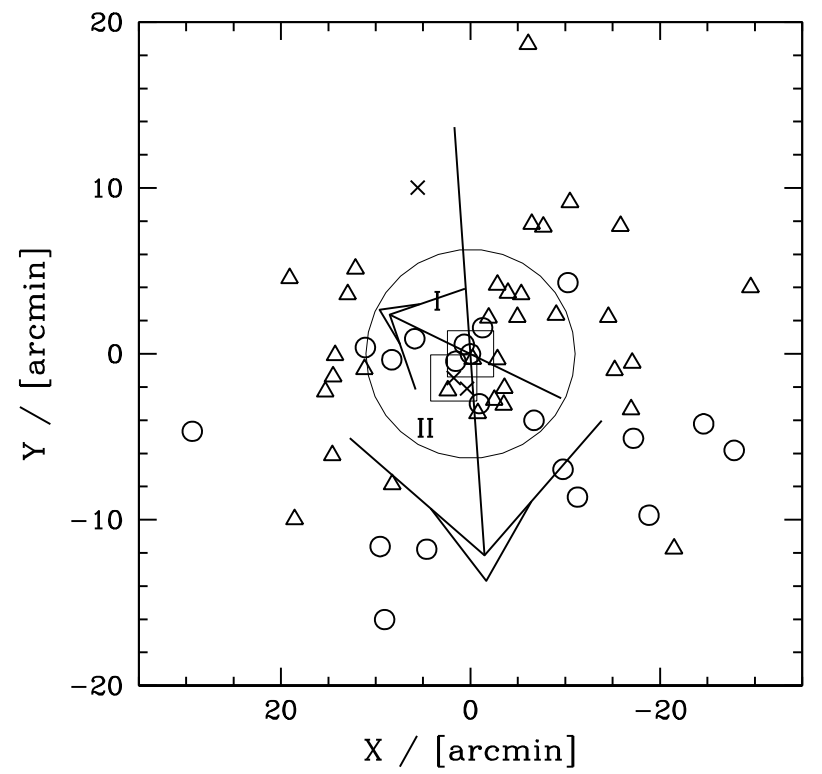

Fig. 7. A610: spatial distribution on the sky of the 57 cluster members. KMM1, KMM2 and KMM3 galaxies are denoted by crosses, triangles and circles, respectively. The position of the two brightest galaxies (BCMI and BCMII) are indicated by the faint large squares. The circle indicates the likely virialized region. Bigger and smaller arrows indicate the velocity gradients within the whole and the virialized regions, respectively. The plot is centered on the cluster center defined as the position of BCMI.

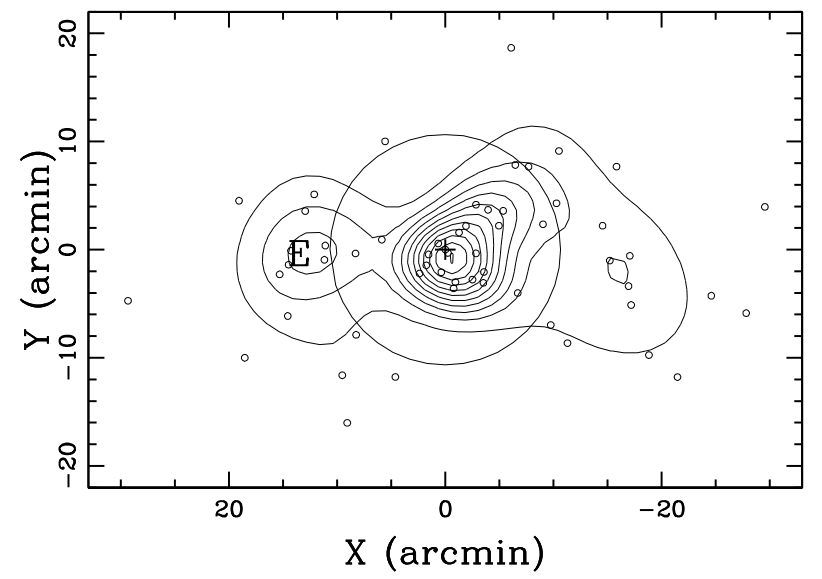

Fig. 8. A610: spatial distribution on the sky of spectroscopically confirmed cluster members and the relative isodensity contour map. The brightest cluster member BCMI is indicated by a cross. The circle indicates the likely virialized region. The Eastern peak is indicated, too. The plot is centered on the cluster center defined as the position of BCMI.

The color-magnitude relation (hereafter CMR), which indicates the early-type galaxy locus, is usually applied to select likely cluster members (see, e.g., A725 in this paper and references therein). When more than two colors are available, it is more effective to select galaxies in color-color space. Here we consider the $i^{\prime}-z^{\prime}$ vs. $r^{\prime}-i^{\prime}$ plane where a rectangular box seems suitable for galaxy selection (e.g., Fig. 12 by Goto et al. 2002). In particular, we consider likely members galaxies having $r^{\prime}$ within $0.08 \mathrm{mag}$ from $r^{\prime}-i^{\prime}=0.41$ and $i^{\prime}-z^{\prime}=0.32$, i.e., the median values recovered from the spectroscopically cluster members (see Fig. 9). The value of $0.08 \mathrm{mag}$ is about two times the typical scatter reported by Goto et al. (2002) for the two CMRs $r^{\prime}-i^{\prime}$ vs. $r^{\prime}$ and $i^{\prime}-z^{\prime}$ vs. $r^{\prime}$. To avoid contamination 


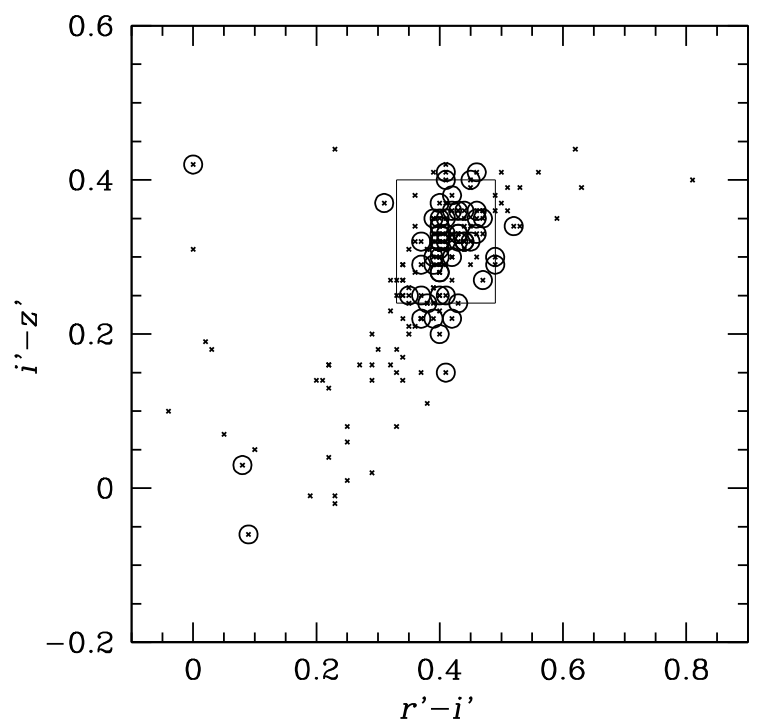

Fig. 9. A610: $i^{\prime}-z^{\prime}$ vs. $r^{\prime}-i^{\prime}$ diagram for galaxies with available spectroscopy is shown by small crosses. The faint square is centered on the median value for colors of member galaxies (circles) and encloses galaxies having colors in the range of 0.08 mag from median values.

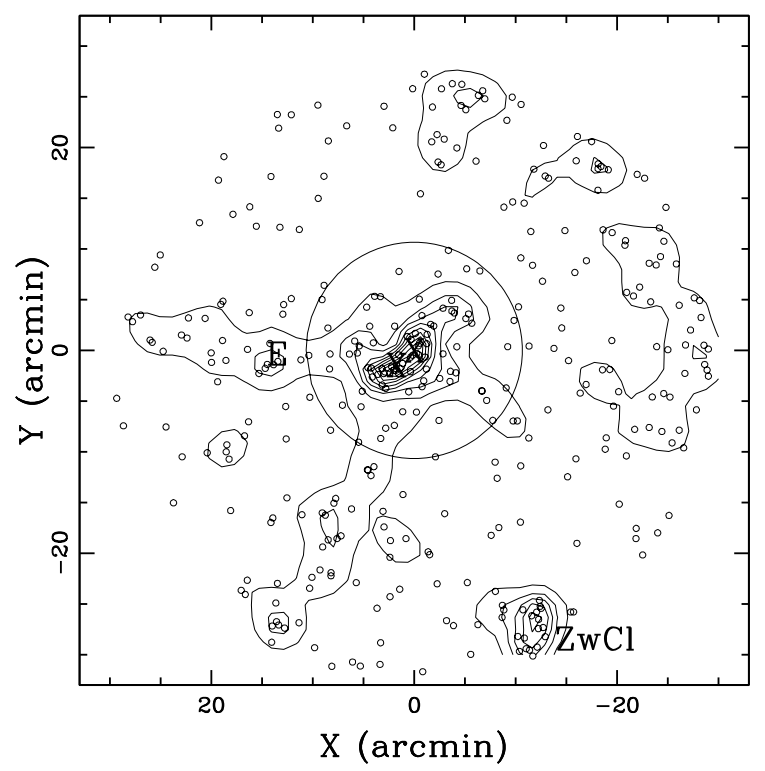

Fig. 10. A610: spatial distribution on the sky and relative isodensity contour map of the 357 likely cluster members (according to the colorcolor diagram) with $r^{\prime} \leq 20$, obtained with the DEDICA method. BCMI and BCMII are indicated by crosses. The circle indicates the likely virialized region. The Eastern peak and the $\mathrm{Zw} \mathrm{Cl0755.2+2649}$ are indicated, too.

by field galaxies we do not show results for galaxies fainter than 20 mag (in $r^{\prime}$-band).

The contour map for the 357 likely cluster members having $r^{\prime} \leq 20$ is shown in Fig. 10. We also analyze galaxies likely members having $r^{\prime} \leq 19$ and $19<r^{\prime} \leq 20$ in a separate way. Our general results are the following. We confirm the presence of the eastern peak, which is generated by bright galaxies. The main structure, contained in the virialized region, shows a much more complex structure having two peaks centered around BCMII and BCMI.

Finally, we notice that the southern peak detected at the southern border of the field is Zwicky Cluster 0755.2+2649

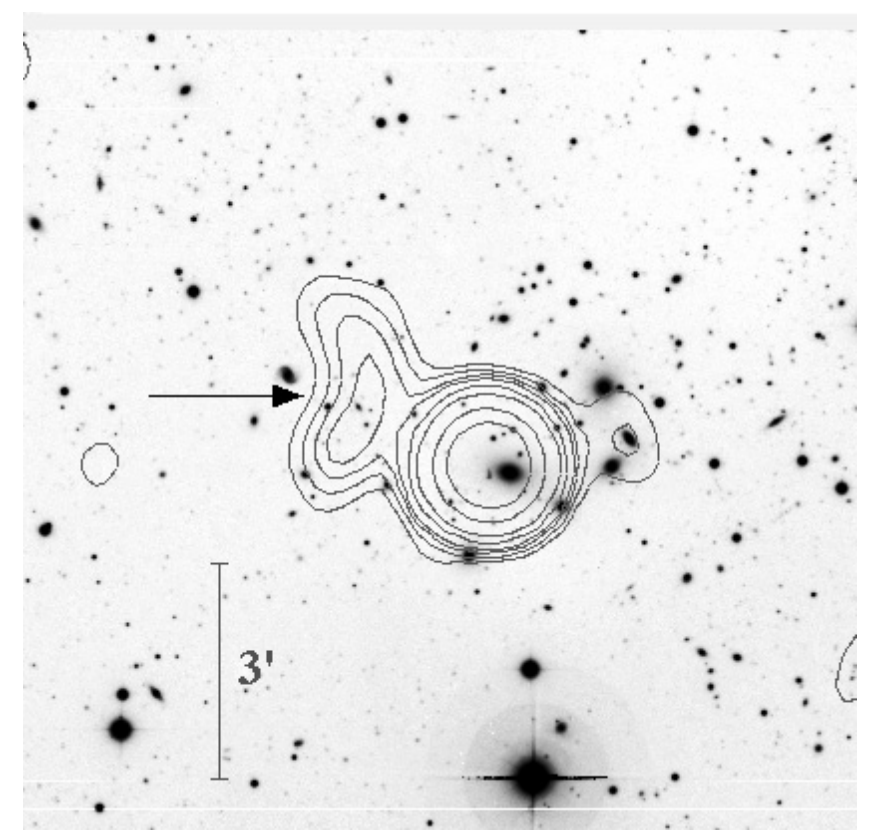

Fig. 11. $R$-band image of A725 (data taken with the WFC camera of the INT) with, superimposed, the contour levels of a radio image from the Westerbork Northern Sky Survey (WENSS, see Kempner \& Sarazin 2001). The arrow shows the position of the radio relic. North is at the top and East to the left.

(recognized by NED as the RXCJ 0758.3 at $z=0.2315$, Böhringer et al. 2000).

\section{Abell 725}

A725 is a poor Abell cluster (Abell richness class $=0$ ). It is characterized by an irregular/clumpy galaxy distribution and a low X-ray luminosity $L_{\mathrm{X}}(0.1-2.4 \mathrm{keV})=0.80 \times 10^{44} h_{50}^{-2} \mathrm{erg} \mathrm{s}^{-1}$ (Böhringer et al. 2000 in their cosmology).

The brightest radio source in the cluster (see Fig. 11) is associated with the bright elliptical in the cluster center at $z=$ $0.0900 \pm 0.0002$ (Owen et al. 1993, 1995). The relic is seen as an arc of diffuse emission to the northeast of this source (Kempner \& Sarazin 2001). Kempner \& Sarazin also noticed that the X-ray gas as seen in the ROSAT All-Sky Survey is slightly elongated along the axis connecting the relic and the cluster center.

Table 4 lists our velocity catalog and Fig. 12 shows the finding chart for the 51 galaxies in the field of A725. In Table 4, for each galaxy, we provide: identification number ID (Col. 1), right ascension and declination, $\alpha$ and $\delta$ (J2000, Col. 2); $R$ and $B$ Johnson magnitudes (Cols. 3 and 4); heliocentric radial velocities, $v=c z_{\odot}(\mathrm{Col} .5)$ with errors, $\Delta v$ (Col. 6). The typical error on the radial velocity as given by the median value is $68 \mathrm{~km} \mathrm{~s}^{-1}$.

\subsection{Member selection and analysis}

Out of 51 galaxies in the sample, the DEDICA method detects A725 as a one-peaked structure, populated by 36 galaxies with $25973 \leq v \leq 29360 \mathrm{~km} \mathrm{~s}^{-1}$ (see Fig. 13). Out of the 15 non-member galaxies, 5 and 10 are foreground and background galaxies, respectively.

As for the cluster center, we consider the position of the brightest cluster member (BCMI; ID 24 in the velocity cata$\log$ ), which was identified with the radio galaxy producing the brightest radio source (see above). No galaxy is rejected by the 


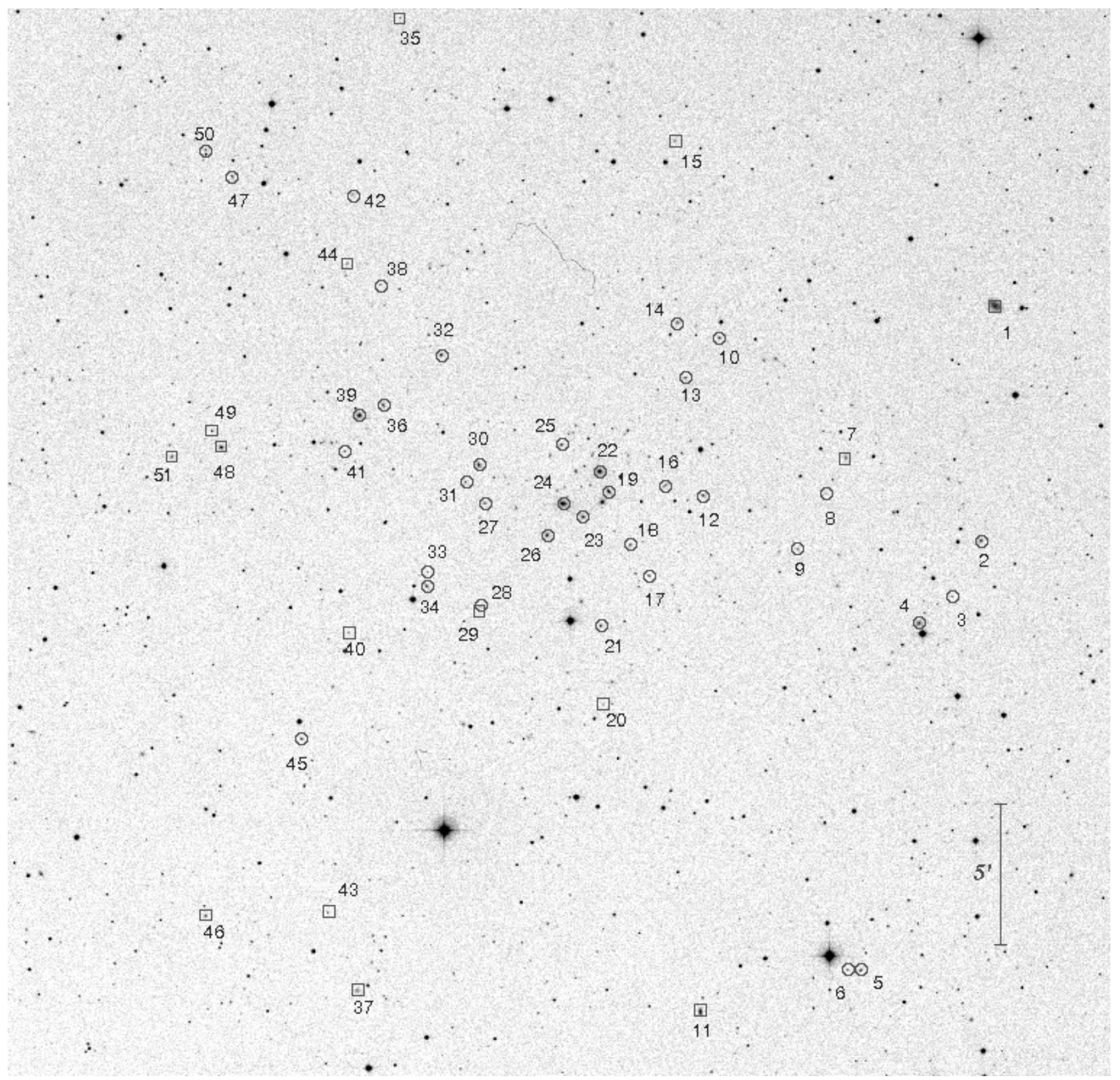

Fig. 12. DSS2 R-band image of A725 (North at the top and East to the left). Galaxies with successful velocity measurements are labeled as in Table 4. Circles and boxes indicate cluster members and non-member galaxies, respectively.

"shifting gapper" leading to a sample of 36 cluster members (see Table 4 and Fig. 14).

By applying the biweight estimators to cluster members (Beers et al. 1990), we compute a mean cluster redshift of $\langle z\rangle=0.0917 \pm 0.0003$, i.e., $\langle v\rangle=27478 \pm 90 \mathrm{~km} \mathrm{~s}^{-1}$ and $\sigma_{\mathrm{V}}=534_{-97}^{+132} \mathrm{~km} \mathrm{~s}^{-1}$. Assuming that the system is in dynamical equilibrium we compute for the radius of the virialized region $R_{\mathrm{vir}}=1.24 h_{70}^{-1} \mathrm{Mpc}$ and $M\left(<R_{\mathrm{vir}}=1.24 h_{70}^{-1} \mathrm{Mpc}\right)=$ $3.2_{-1.2}^{+1.6} \times 10^{14} h_{70}^{-1} M_{\odot}$.

The values of the kurtosis (3.998) and the scaled tail index (1.504) indicate that the velocity distribution is lightertailed than a Gaussian at the 90-95\% and the 95-99\% c.l.s (see Table 2 of Bird \& Beers 1993), respectively. The BCMI shows evidence of peculiarity, according to the Indicator test by Gebhardt \& Beers (1991, at the $>95 \%$ c.l.), with respect to the whole system. We do not find any other trace of substructure.
In this cluster, we find evidence of luminosity segregation. For the 33 galaxies having available magnitude, the magnitude $R$ correlates with the clustercentric distance at the $99.5 \%$ c.l., according to the Spearman correlation coefficient.

\section{2. $2 D$ galaxy distribution}

By applying the DEDICA method to the 2D distribution of A725 cluster members we find that the cluster is elongated along the EW direction.

We recover our photometric catalog selecting likely members on the basis of the $B-R$ vs. $R$ relation (see, e.g., Barrena et al. 2007b). To determine the relation we fix the slope according to López-Cruz et al. (2004, see their Fig. 3) and apply the two-sigma-clipping fitting procedure to the cluster members obtaining $B-R=2.971-0.054 \times R$. In our photometric catalog we 


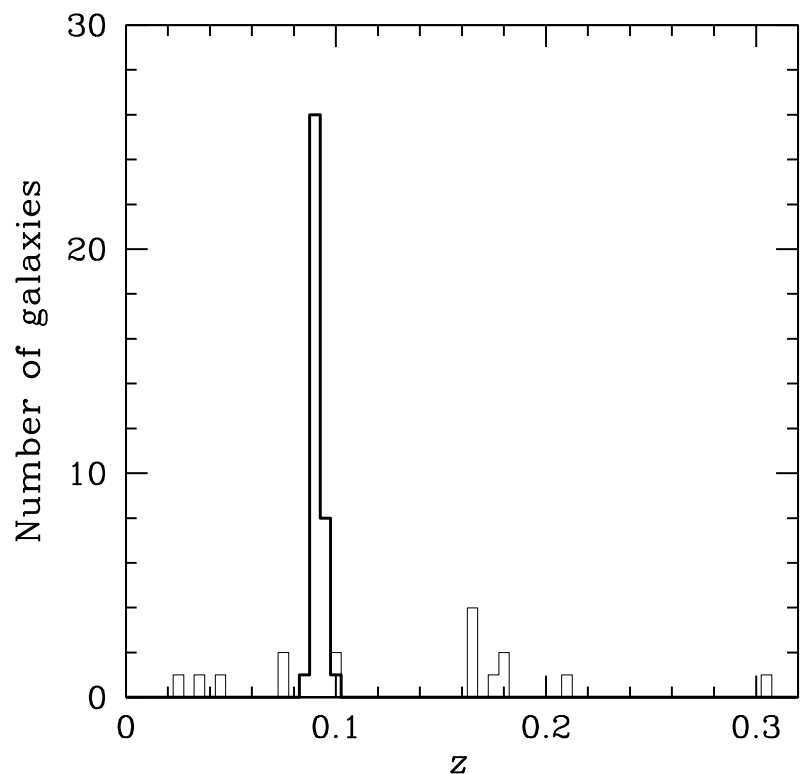

Fig. 13. A725: redshift galaxy distribution. The solid line histogram refers to galaxies assigned to the cluster according to the DEDICA reconstruction method.

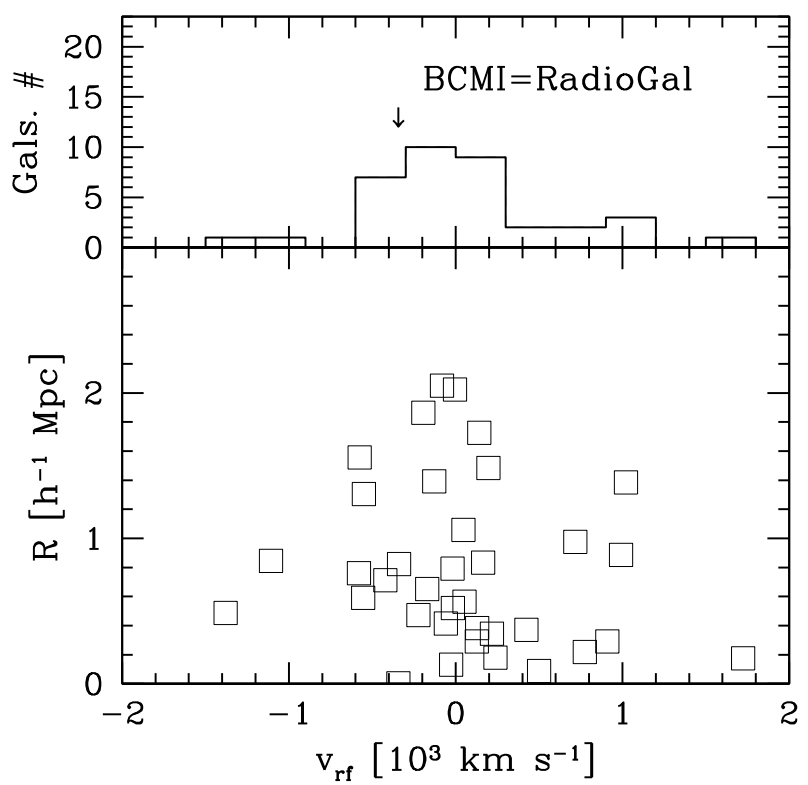

Fig. 14. A725: Bottom panel: rest-frame velocity vs. projected clustercentric distance for the 36 member galaxies. Top panel: velocity distribution of 36 cluster members. The arrow corresponds to the brightest cluster member.

consider galaxies (objects with SExtractor stellar index $\leq 0.9$ ) lying within $0.25 \mathrm{mag}$ of the relation. To avoid contamination by field galaxies we do not show results for galaxies fainter than 20 (in the $R$-band).

The contour map for the 360 likely cluster members having $R \leq 20$ is elongated along the E-W direction in the central region and the position of the density peak lies about $2^{\prime}$ from the BCMI, assumed as cluster center (see Fig. 15). Moreover, the cluster shows an important secondary peak towards NE and the cluster itself appear elongated along the NE-SW direction (see the isodensity contours at the virial radius distance). Similar features are obtained analyzing other magnitudes cuts $(R \leq 19$ or $R \leq 18$ ).

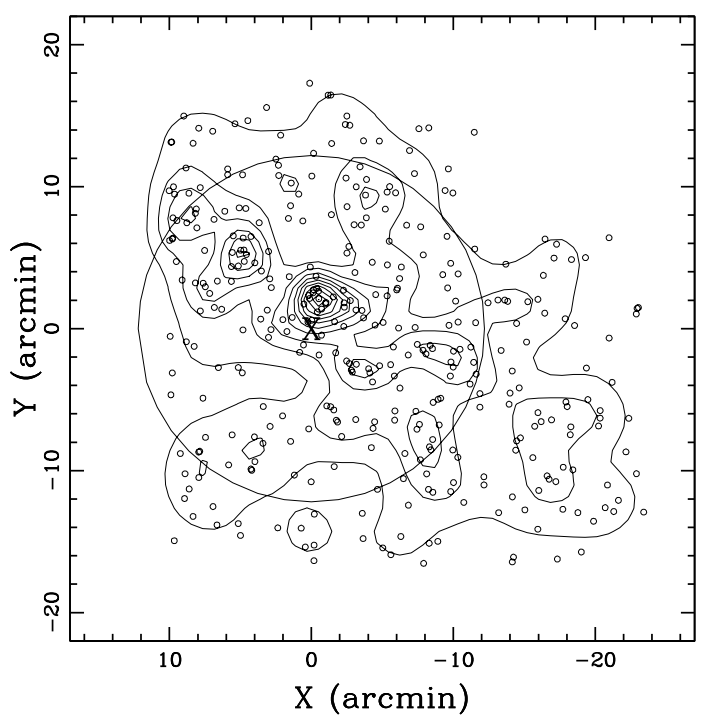

Fig. 15. A725: spatial distribution on the sky and relative isodensity contour map of the 360 likely cluster members (according to the two colors selection) with $R \leq 20$, obtained with the DEDICA method. The cross indicates the BCMI. The circle indicates the likely virialized region.

\section{Abell 796}

A796 is an Abell cluster of Abell richness class = 1. It is characterized by a clumpy galaxy distribution and a low X-ray luminosity $L_{\mathrm{X}}(0.1-2.4 \mathrm{keV})=1.38 \times 10^{44} h_{50}^{-2} \mathrm{erg} \mathrm{s}^{-1}($ Kempner \& Sarazin 2001 in their cosmology).

Kempner \& Sarazin (2001) found a centrally-located diffuse radio emission which is quite large and has a very low surface brightness. When converting in our cosmology the X-ray luminosity and the radio halo power by Kempner \& Sarazin (2001), we find $L_{X}(0.1-2.4 \mathrm{keV})=0.93 \times 10^{44} h_{70}^{-2} \mathrm{erg} \mathrm{s}^{-1}$ and $P_{1.4 \mathrm{GHz}}=(5.6 \pm 2.1) \times 10^{23} \mathrm{~W} \mathrm{~Hz}^{-1}$, respectively. These nominal values stay far from the correlation between radio power and X-ray luminosity as given by Cassano et al. (2006, see their Fig. 1). However, the halo is uncertain and the error on the radio power is so large that deeper imaging is needed to confirm the presence of the radio halo and to measure its flux with greater precision. Owen et al. (1993) found two close radio galaxies in the field of this cluster. The radio source $\mathrm{A}$ is here identified with a background AGN (ID 65 at $z \sim 0.295$ in our catalog, see below), while we have no $z$ for the radio source $B$.

Table 5 lists our velocity catalog, and Fig. 16 shows the finding chart for the 99 galaxies in the field of A796. In Table 5, for each galaxy we provide: identification number ID (Col. 1), right ascension and declination, $\alpha$ and $\delta$ (J2000, Col. 2); $r^{\prime}$ SDSS magnitudes (Col. 3); heliocentric radial velocities, $v=c z_{\odot}$ (Col. 4) with errors, $\Delta v$ (Col. 5); source of velocity data (W: WHT, S: SDSS, W+S: combined WHT and SDSS) in Col. 6. The typical error on radial velocity as given by the median value is $45 \mathrm{~km} \mathrm{~s}^{-1}$.

\subsection{Member selection and analysis}

Out of 99 galaxies in the sample, the DEDICA method detects A796 as a one-peaked structure, populated by 27 galaxies with $45242 \leq v \leq 50023 \mathrm{~km} \mathrm{~s}^{-1}$ (see Fig. 17). Out of the nonmember galaxies, 38 and 34 are foreground and background galaxies, respectively. 


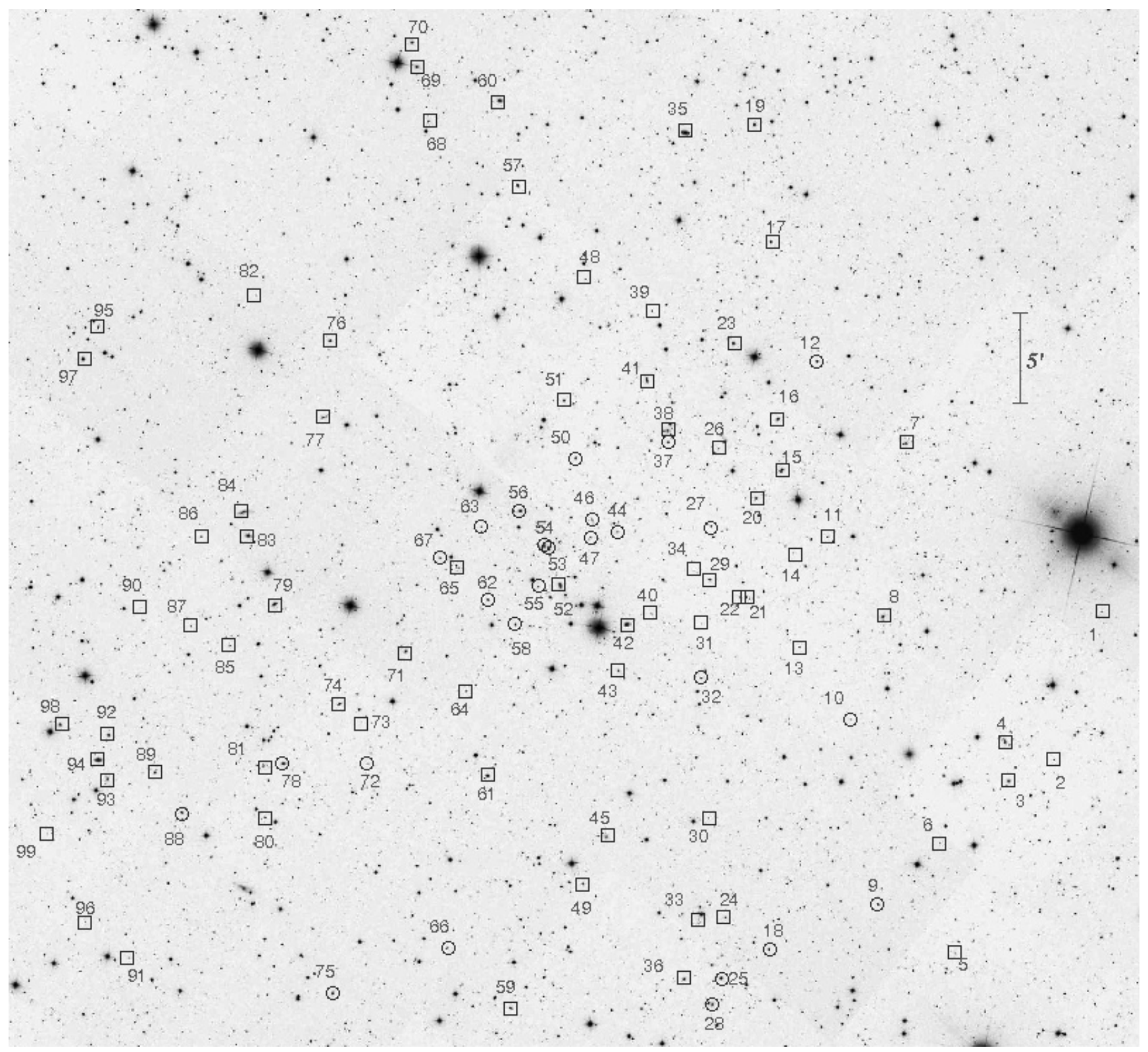

Fig. 16. SDSS image of A796 (North at the top and East to the left). Galaxies with successful velocity measurements are labeled as in Table 5. Circles and boxes indicate cluster members and non-member galaxies, respectively.

As for the cluster center, we consider the position of the peak in the 2D galaxy distribution as determined by the 2D DEDICA method $\left(\mathrm{RA}=09^{\mathrm{h}} 27^{\mathrm{m}} 57^{\mathrm{s}} .55, \mathrm{Dec}=+60^{\circ} 26^{\prime} 33^{\prime \prime}\right.$. 7 (J2000)]. Only one galaxy is then rejected by the "shifting gapper" leading to a sample of 26 cluster members (see Table 5 and Fig. 18).

Figure 18 also shows the velocity (with respect to the cluster mean) of the two luminous cluster galaxies, IDs 56 and 54. They have a comparable $r^{\prime}$ magnitude, but while ID 54 lies somewhat closer to the cluster center, ID 56 lies $\lesssim 0.4 h_{70}^{-1} \mathrm{Mpc}$ from the cluster center. Hereafter, we refer to IDs 56 and 54 as BCMInc and BCMI, respectively.

By applying the biweight estimators to 26 cluster members (Beers et al. 1990), we compute a mean cluster redshift of $\langle z\rangle=0.1566 \pm 0.0005$, i.e., $\langle v\rangle=46942 \pm 140 \mathrm{~km} \mathrm{~s}^{-1}$ and $\sigma_{\mathrm{V}}=698_{-159}^{+216} \mathrm{~km} \mathrm{~s}^{-1}$. Assuming that the system is in dynamical equilibrium we compute for the radius of the virialized region $R_{\mathrm{vir}}=1.57 h_{70}^{-1} \mathrm{Mpc}$ and the virial mass $M\left(<R_{\mathrm{vir}}=\right.$ $\left.1.57 h_{70}^{-1} \mathrm{Mpc}\right)=6.9_{-3.0}^{+4.0} \times 10^{14} h_{70}^{-1} M_{\odot}$.
We also consider an alternative sample of 24 galaxies Sample 2 - rejecting the two galaxies with the highest velocity that are separated by a gap of $\sim 820 \mathrm{~km} \mathrm{~s}^{-1}$ in the reference frame from the whole velocity distribution of the cluster (see Fig. 18). This gap is detected with a significance level of (1-3.0E-2) in the velocity distribution (see Fig. 19). The properties of Sample2 are listed in Table 6.

We also present the analysis of the 14 galaxies which are the subsample of Sample2 contained within $R_{\text {vir }}$ (hereafter the "virialized subsample") to consider galaxies likely belonging to the true internal, virialized structure.

Considering the whole sample of 26 galaxies we find the presence of a velocity gradient at the $98.9 \%$ c.l., with high velocity galaxies SSE located $\left(\mathrm{PA}=156_{-26}^{+21}\right.$ ). Accordingly, the two subsamples of galaxies having lower or higher velocity than the median velocity show different distributions in the sky (at the $99.5 \%$ according to the 2DKS test). Moreover, the DresslerSchectman test finds substructure at the $96 \%$ c.l. However, these 


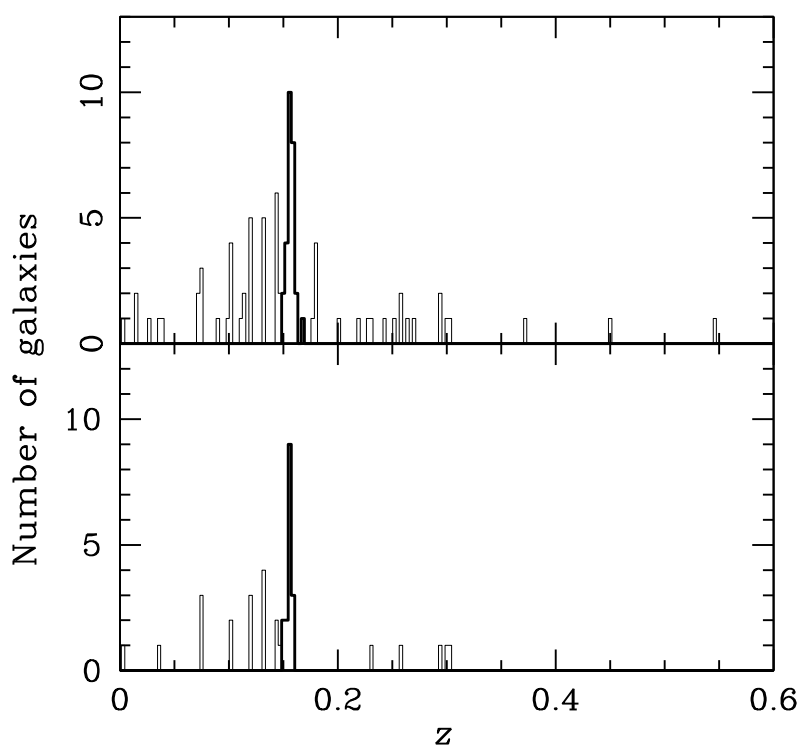

Fig. 17. A796: redshift galaxy distribution of only galaxies with $z<0.6$. Top panel. Whole catalog: the solid line histogram refers to galaxies assigned to the cluster according to the DEDICA reconstruction method. Bottom panel. The same for galaxies within $15^{\prime}$ from the cluster center.

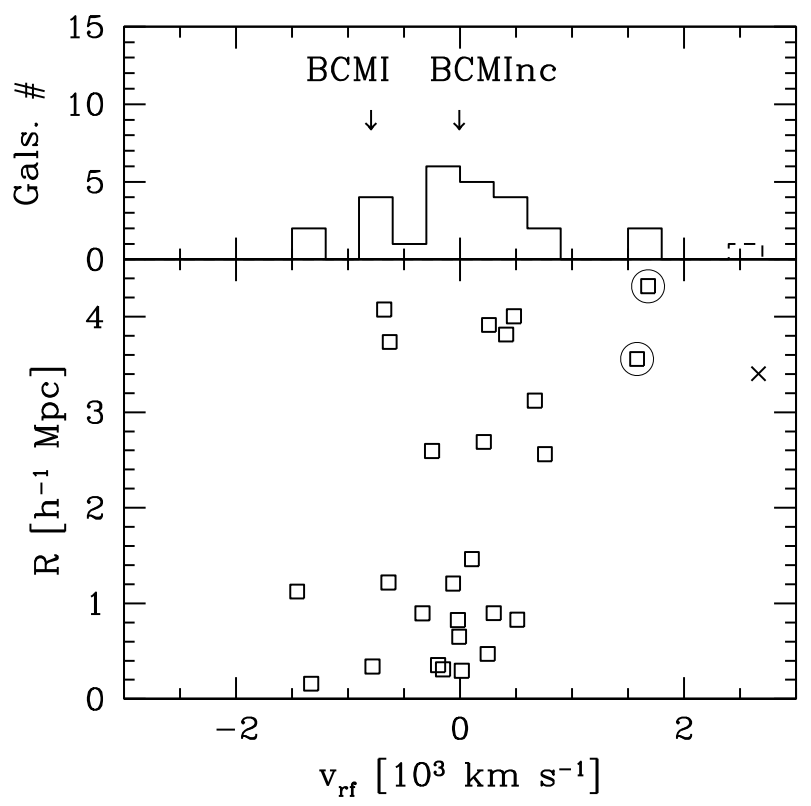

Fig. 18. A796: Bottom panel: rest-frame velocity vs. projected clustercentric distance for the 27 galaxies in the main peak (Fig. 3) showing galaxies detected as interloper by our "shifting gapper" procedure (crosses). Squares indicate galaxies forming the sample of member galaxies. The two galaxies with the highest velocities are rejected to build Sample2 (see text). Top panel: velocity distribution of the 26 cluster members and the rejected galaxy (solid and dashed histograms). Arrows correspond to the two luminous member galaxies.

pieces of evidence of correlation between velocities and positions are no longer detected when we analyze Sample2 and the "virialized sample". Thus, we suspect that the correlations found are not really due to cluster structure, but rather to the large-scale structure environment. On the other hand the direction of the velocity gradient coincides with that shown by the cluster elongation in the 2D analysis (see in the following). In all our three samples, the BCMI shows evidence of peculiarity according to

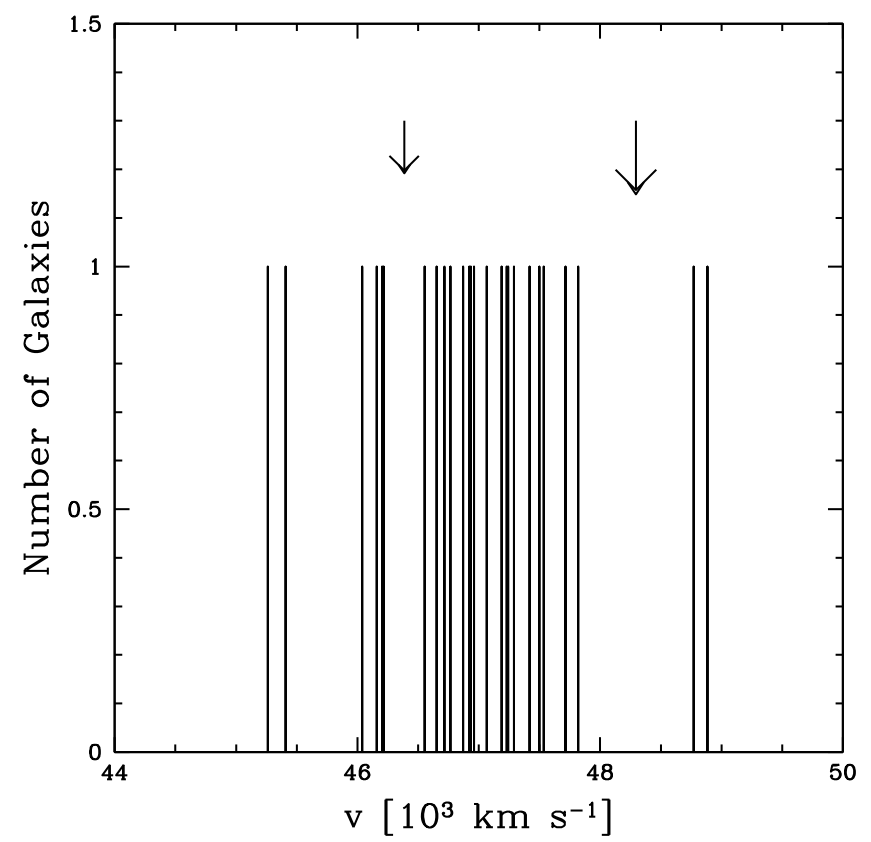

Fig. 19. A796: stripe density plot where the big (small) arrow indicates the position of the significant gap in the velocity distribution of the whole system (Sample2).

Table 6. A796: results of the kinematical analysis.

\begin{tabular}{lccccc}
\hline \hline Sample & $N_{\mathrm{g}}$ & $\begin{array}{c}\langle v\rangle \\
\mathrm{km} \mathrm{s}^{-1}\end{array}$ & $\begin{array}{c}\sigma_{\mathrm{V}} \\
\mathrm{km} \mathrm{s}^{-1}\end{array}$ & $\begin{array}{c}R_{\text {vir }} \\
\mathrm{Mpc}\end{array}$ & $\begin{array}{c}\text { Mass }\left(<R_{\text {vir }}\right) \\
10^{14} M_{\odot}\end{array}$ \\
\hline Whole system & 26 & $46942 \pm 140$ & $698_{-159}^{+216}$ & 1.57 & $6.9_{-3.0}^{+4.0}$ \\
Whole system Sample2 & 24 & $46921 \pm 120$ & $571_{-73}^{+134}$ & 1.29 & $3.9_{-2.2}^{+2.4}$ \\
Virialized subsample & 14 & $46796 \pm 165$ & $586_{-148}^{+171}$ & 1.32 & $4.6_{-1.6}^{+2.4}$ \\
\hline
\end{tabular}

the Indicator test by Gebhardt \& Beers (1991, at the $>95 \%$ c.l.) with respect to the whole system.

Considering Sample2, we find a weighted gap which separates the velocity distribution in a low and a high velocity set (see Fig. 19). Very interestingly, the two most luminous galaxies BCMI and BCMInc lie in the low and high velocity sets, respectively. This is suggestive of a situation similar to that of A610, i.e., a possible merger between two subclusters.

\section{2. $2 D$ galaxy distribution}

When applying the DEDICA method to the 2D distribution of A796 galaxy members we find that the cluster is elongated along the SE-NW direction. To check this finding we recover the photometric catalog extracted from the SDSS DR5. Figure 20 shows the result of the DEDICA method applied to the $r^{\prime} \leq 20$ likely members selected on the basis of their colors - i.e., only galaxies lying within $0.08 \mathrm{mag}$ from $r^{\prime}-i^{\prime}=0.46$ and $i^{\prime}-z^{\prime}=0.33$ colors. We confirm the cluster elongation finding two minor peaks in the SE and NW directions. Similar results are found for different magnitude cuts $r^{\prime} \leq 19$ and $r^{\prime} \leq 21$.

\section{Summary and conclusions}

We present the results of a dynamical analysis of the three poor, low X-ray luminous clusters A610, A725, and A796 containining diffuse radio sources (relic, relic, and a possible halo, respectively). Our analysis is based on new redshift data for 
Table 7. Properties of galaxy clusters.

\begin{tabular}{lrrrcccc}
\hline \hline Cluster & $N$ & $N_{\mathrm{m}}$ & $N_{\mathrm{m}, \text { vir }}$ & $\begin{array}{c}\alpha \\
\mathrm{J} 2000\end{array}$ & $z$ & $\begin{array}{c}\sigma_{\mathrm{V}} \\
\mathrm{km} \mathrm{s}^{-1}\end{array}$ & $\begin{array}{c}\text { Mass }\left(<R_{\text {vir }}\right) \\
10^{14} M_{\odot}\end{array}$ \\
\hline A610 & 165 & 57 & 22 & $075917.10+270916.1$ & 0.098 & $426-496$ & $1.8-2.3$ \\
A725 & 51 & 36 & 27 & $090109.99+623720.0$ & 0.092 & $\sim 534$ & $\sim 3.2$ \\
A796 & 99 & 26 & 14 & $092757.55+602633.7$ & 0.157 & $571-698$ & $3.9-6.9$ \\
\hline
\end{tabular}

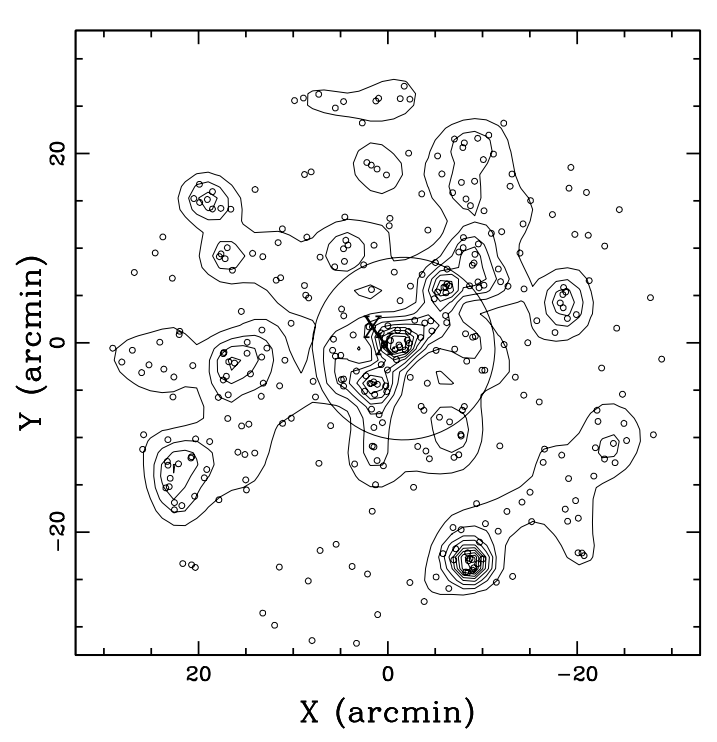

Fig. 20. A796: spatial distribution on the sky and relative isodensity contour map of the 349 likely cluster members (according to the two colors selection) with $r^{\prime} \leq 20$, obtained with the DEDICA method. The circle indicates the likely virialized region. The crosses indicate the two most luminous galaxies BCMI and BCMInc.

158 galaxies obtained at the WHT and additional SDSS DR5 data for A610 and A796. We also use new photometric data obtained at the INT for A725.

We select 57, 36 and 26 cluster members for A610, A725 and A796, respectively. Out of these galaxies 35, 36 and 17 were measured by the WHT.

The low values we compute for the global LOS velocity dispersion of galaxies $\left(\sigma_{\mathrm{V}}=420-700 \mathrm{~km} \mathrm{~s}^{-1}\right)$ confirm that these clusters are low-mass clusters. Table 7 summarizes the main cluster properties as recovered in this study. Using the $\sigma_{\mathrm{V}}-T_{\mathrm{X}}$ relation by Girardi et al. (1998) the range of expected X-ray temperature of the intracluster gas is $T_{\mathrm{X}}=2-3 \mathrm{keV}$. The study of these three clusters indicates that the phenomenon of diffuse radio sources is not limited to massive clusters only.

We also discuss the dynamical status and the possible connection with the diffuse sources of each individual cluster. Regarding the two clusters hosting a radio relic, A610 and A725, the former is better sampled and can be analyzed in greater detail. A610 shows a lot of evidence of substructure: the nonGaussianity of the velocity distribution, the correlation between galaxy velocities and positions and the peculiarity of the BCMI in the velocity space. A610 seems formed by two structures separated by $\sim 700 \mathrm{~km} \mathrm{~s}^{-1}$ in the cluster rest-frame, having comparable $\sigma_{\mathrm{V}} \sim 200 \mathrm{~km} \mathrm{~s}^{-1}$ and likely causing a velocity gradient. Moreover, the BCMI velocity is very close to the mean velocity of the higher velocity structure. A third small, low velocity group hosts the BCMII. The analysis of the 2D galaxy distribution shows a bimodal distribution in the core - elongated in the SE-NW direction and likely associated to BCMI and BCMII groups - as well as the presence of an eastern group just outside the virialized region. In this chaotic scenario, it is not easy to unveil the event responsible for the relic. One possibility is that the relic be associated to the likely merger between the two structures corresponding to BCMI and BCMII. In this case it would be somewhat perpendicular to the axis connecting the two merging structures (see Fig. 1) in agreement with being originated by shock waves connected to the ongoing merger (e.g., Ensslin \& Brüggen 2002). Another possibility is that the relic be related to the merger between the two main velocity structures forming the cluster (see Fig. 4). This would be more likely from the energetic point of view since this merger involves structures of 1:1 mass ratio. In conclusion, A610 represents another case of a radio cluster formed by two merging subclusters, like, e.g., A115 (Barrena et al. 2007b); A2744 (Boschin et al. 2006); and A773 (Barrena et al. 2007a), but at much smaller scales (global $\sigma_{\mathrm{V}} \sim 500 \mathrm{~km} \mathrm{~s}^{-1}$ vs. $\sigma_{\mathrm{V}}>1000 \mathrm{~km} \mathrm{~s}^{-1}$ ). As a further support to the merging scenario, we notice that the BCMI of A610 is also a bright radio source with a WAT structure. This is very interesting because WATs are only found in galaxy clusters and are excellent probes of the gas-dynamical processes occuring during a cluster merger (e.g., Gómez et al. 1997).

As for A725, it shows some evidence of non-Gaussianity in the velocity distribution, the peculiarity of the BCMI in the velocity space, and an elongated cluster shape in the NE-SW direction. This direction is the same one indicated by the radio relic since it is an arc of diffuse emission to the NE of the radio galaxy associated with the BCMI (Kempner \& Sarazin 2001, see Fig. 11).

A796 possibly hosts a radio halo (to be confirmed) according to WENSS data (Kempner \& Sarazin 2001). It shows the peculiarity of the BCMI in the velocity space, possible spatial/velocity substructure and the presence of two minor clumps along the SE-NW direction.

For A725 and A796 the number of cluster members ( 30) is enough for the estimate of $\sigma_{\mathrm{V}}$, but too small for the analysis of substructure. Thus, a definitive conclusion will require more data.

Acknowledgements. We would like to thank Luigina Feretti for many enlightening discussions and for the VLA radio image of A610 she kindly provided us. We thank the anonymous referee for useful comments and suggestions.

This publication is based on observations made on the island of La Palma with the William Herschel Telescope (WHT) and with the Isaac Newton Telescope (INT), operated by the Isaac Newton Group (ING), in the Spanish Observatory of the Roque de Los Muchachos of the Instituto de Astrofisica de Canarias.

This research has made use of the NASA/IPAC Extragalactic Database (NED), which is operated by the Jet Propulsion Laboratory, California Institute of Technology, under contract with the National Aeronautics and Space Administration.

This research has made use of the galaxy catalog of the Sloan Digital Sky Survey (SDSS). Funding for the SDSS has been provided by the Alfred P. Sloan Foundation, the Participating Institutions, the National Aeronautics and Space Administration, the National Science Foundation, the U.S. Department of Energy, the Japanese Monbukagakusho, and the Max Planck Society. The SDSS Web site is http: //www. sdss.org/.

The SDSS is managed by the Astrophysical Research Consortium for the Participating Institutions. The Participating Institutions are the American Museum of Natural History, Astrophysical Institute Potsdam, University of Basel, University of Cambridge, Case Western Reserve University, University 
of Chicago, Drexel University, Fermilab, the Institute for Advanced Study, the Japan Participation Group, Johns Hopkins University, the Joint Institute for Nuclear Astrophysics, the Kavli Institute for Particle Astrophysics and Cosmology, the Korean Scientist Group, the Chinese Academy of Sciences (LAMOST), Los Alamos National Laboratory, the Max-Planck-Institute for Astronomy (MPIA), the Max-Planck-Institute for Astrophysics (MPA), New Mexico State University, Ohio State University, University of Pittsburgh, University of Portsmouth, Princeton University, the United States Naval Observatory, and the University of Washington.

This work was partially supported by a grant from the Istituto Nazionale di Astrofisica (INAF, grant PRIN-INAF2006 CRA ref number 1.06.09.06).

\section{References}

Abell, G. O., Corwin, H. G. Jr., \& Olowin, R. P. 1989, ApJS, 70, 1 Ashman, K. M., Bird, C. M., \& Zepf, S. E. 1994, AJ, 108, 2348

Bardelli, S., Zucca, E., Vettolani, G., et al. 1994, MNRAS, 267, 665

Barrena, R., Biviano, A., Ramella, M., Falco, E. E., \& Seitz, S. 2002, A\&A, 386, 816

Barrena, R., Boschin, W., Girardi, M., \& Spolaor, M. 2007a, A\&A, 467, 37

Barrena, R., Boschin, W., Girardi, M., \& Spolaor, M. 2007b, A\&A, 469, 861

Beers, T. C., Flynn, K., \& Gebhardt, K. 1990, AJ, 100, 32

Beers, T. C., Forman, W., Huchra, J. P., Jones, C., \& Gebhardt, K. 1991, AJ, 102, 1581

Bertin, E., \& Arnouts, S. 1996, A\&AS, 117, 393

Bird, C. M. 1994, ApJ, 422, 480

Bird, C. M., \& Beers, T. C. 1993, AJ, 105, 1596

Böhringer, H., \& Schuecker, P. 2002, in Merging Processes in Galaxy Clusters, ed. L. Feretti, I. M. Gioia, \& G. Giovannini, Observational signatures and statistics of galaxy cluster mergers (The Netherlands: Kluwer Ac. Pub.)

Böhringer, H., Voges, W., Huchra, J. P., et al. 2002, ApJS, 129, 435

Boschin, W., Girardi, M., Barrena, R., et al. 2004, A\&A, 416, 839

Boschin, W., Girardi, M., Spolaor, M., \& Barrena, R. 2006, A\&A, 449, 461

Buote, D. A. 2001, ApJ, 553, L15

Buote, D. A. 2002, in Merging Processes in Galaxy Clusters, ed. L. Feretti, I. M. Gioia, \& G. Giovannini, Optical Analysis of Cluster Mergers (The Netherlands: Kluwer Ac. Pub.)

Burns, J. O., Roettiger, K., Ledlow, M., \& Klypin, A. 1994, ApJ, 427, L87

Burstein, D., \& Heiles, C. 1982, AJ, 87, 1165

Carlberg, R. G., Yee, H. K. C., \& Ellingson, E. 1997, ApJ, 478, 462

Cassano, R., Brunetti G., \& Setti, G. 2006, MNRAS, 369, 1577

Cousins, A. W. J. 1976, MemRAS, 81, 25

Danese, L., De Zotti, C., \& di Tullio, G. 1980, A\&A, 82, 322

den Hartog, R., \& Katgert, P. 1996, MNRAS, 279, 349

Ellingson, E., \& Yee, H. K. C. 1994, ApJS, 92, 33

Ensslin, T. A., \& Brüggen, M. 2002, MNRAS, 331, 1011

Fadda, D., Girardi, M., Giuricin, G., Mardirossian, F., \& Mezzetti, M. 1996, ApJ, 473, 670

Feretti, L. 1999, MPE Rep., 271

Feretti, L. 2002, The Universe at Low Radio Frequencies, held 30 Nov.-4 Dec. 1999, Pune, India, ed. A. Pramesh Rao, G. Swarup, \& Gopal-Krishna, Proc. IAU Symp., 199, 133

Feretti, L. 2005, X-Ray and Radio Connections, Published electronically by NRAO, http://www.aoc.nrao.edu/events/xraydio, Held 3-6 February 2004 in Santa Fe, New Mexico, USA, ed. L. O. Sjouwerman, \& K. K. Dyer

Gebhardt, K., \& Beers, T. C. 1991, ApJ, 383, 72
Giovannini, G., \& Feretti, L. 2000, New Astron., 5, 335

Giovannini, G., \& Feretti, L. 2002, in Merging Processes in Galaxy Clusters, ed. L. Feretti, I. M. Gioia, \& G. Giovannini, Diffuse Radio Sources and Cluster Mergers (The Netherlands: Kluwer Ac. Pub.)

Giovannini, G., Feretti, L., Venturi T., Kim, K.-T., \& Kronberg, P. P. 1993, ApJ, 406, 399

Giovannini, G., Tordi, M., \& Feretti, L. 1999, New Astron., 4, 141

Girardi, M., \& Biviano, A. 2002, in Merging Processes in Galaxy Clusters, ed.

L. Feretti, I. M. Gioia, \& G. Giovannini, Analysis of Cluster Mergers (The Netherlands: Kluwer Ac. Pub.)

Girardi, M., \& Mezzetti, M. 2001, ApJ, 548, 79

Girardi, M., Fadda, D., Giuricin, G., et al. 1996, ApJ, 457, 61

Girardi, M., Giuricin, G., Mardirossian, F., Mezzetti, M., \& Boschin, W. 1998, ApJ, 505, 74

Girardi, M., Boschin, W., \& Barrena, R. 2006, A\&A, 455, 45

Gómez, P. L., Pinkney, J., Burns, J. O., et al. 1997, ApJ, 474, 580

Govoni, F., Ensslin, T. A., Feretti, L., \& Giovannini, G. 2001a, A\&A, 369, 441

Govoni, F., Feretti, L., Giovannini, G., et al. 2001b, A\&A, 376, 803

Goto, T., Sekiguchi, M., Nichol, R. C., et al. 2002, AJ, 123, 1807

Gullixson, C. A. 1992, in Astronomical CCD Observing and Reduction Techniques, ed. S. B. Howell, ASP Conf. Ser., 23, 130

Hanisch, R. J. 1982, A\&A, 116, 137

Jetha, N. N., Hardcastle, M. J., \& Sakelliou, I. 2006, MNRAS, 368, 609

Johnson, H. L., \& Morgan, W. W. 1953, ApJ, 117, 313

Kempner, J. C., \& Sarazin, C. L. 2001, ApJ, 548, 639

Kennicutt, R. C. 1992, ApJS, 79, 225

Kowalski, M. P., Ulmer, M. P., Cruddace, R. G., \& Wood, K. S. 1984, ApJS, 56, 403

Limber, D. N., \& Mathews, W. G. 1960, ApJ, 132, 286

López-Cruz, O., Barkhouse, W. A., \& Yee, H. K. C. 2004, ApJ, 614, 679

Malumuth, E. M., Kriss, G. A., Dixon, W. Van Dyke, Ferguson, H. C., \& Ritchie, C. 1992, AJ, 104, 495

Maurogordato, S., Cappi, A., Ferrari, C., et al. 2008, A\&A, in press

Mercurio, A., Girardi, M., Boschin, W., Merluzzi, P., \& Busarello, G. 2003, A\&A, 397, 431

Owen, F. N., White, R. A., \& Burns, J. O. 1992, ApJS, 80, 501

Owen, F. N., White, R. A., \& Jingping, G. 1993, ApJS, 87, 135

Owen, F. N., Ledlow, M. J., \& Keel, W. C. 1995, AJ, 109, 14

Pisani, A. 1993, MNRAS, 265, 706

Pisani, A. 1996, MNRAS, 278, 697

Poggianti, B. M. 1997, A\&AS, 122, 399

Press, W. H., Teukolsky, S. A., Vetterling, W. T., \& Flannery, B. P. 1992, in Numerical Recipes, 2nd Ed. (Cambridge University Press)

Quintana, H., Carrasco, E. R., \& Reisenegger, A. 2000, AJ, 120, 511

Roettiger, K., Loken, C., \& Burns, J. O. 1997, ApJS, 109, 307

Röttgering, H., Snellen, I., Miley, G., et al. 1994, ApJ, 436, 654

Sarazin, C. L. 2002, in Merging Processes in Galaxy Clusters, The Physics of Cluster Mergers, ed. L. Feretti, I. M. Gioia, \& G. Giovannini (The Netherlands: Kluwer Ac. Pub.)

Schuecker, P., Böhringer, H., Reiprich, T. H., \& Feretti, L. 2001, A\&A, 378, 408 Shapiro, S. S., \& Wilk, M. B. 1965, Biometrika, 52, 591

Struble, M. F., \& Rood, H. J. 1982, AJ, 87, 7

Struble, M. F., \& Rood, H. J. 1984, AJ, 89, 1487

The, L. S., \& White, S. D. M. 1986, AJ, 92, 1248

Tonry, J., \& Davis, M. 1979, AJ, 84, 1511

Tribble, P. 1993, MNRAS, 263, 31

Valentijn, E. A. 1979, ApJS, 38, 319

Wainer, H., \& Schacht, S. 1978, Psychometrika, 43, 203 
W. Boschin et al.: Optical analysis of poor radio clusters, Online Material p 1

Table 1. Velocity catalog of 165 spectroscopically measured galaxies in the field of A610. In Col. 1, IDs in italics indicate non-cluster galaxies. IDs 91 and 98 (in boldface) are, respectively, the first and the second brightest galaxies of the cluster. Asterisks in Col. 1 highlight QSOs in the SDSS catalog.

\begin{tabular}{|c|c|c|c|c|c|}
\hline ID & $\alpha, \delta(\mathrm{J} 2000)$ & $r^{\prime}$ & $\begin{array}{l}v \\
(\mathrm{~km}\end{array}$ & & Sourc \\
\hline 1 & $75704.15,+271313.3$ & 17.65 & 29052 & 16 & S \\
\hline 2 & 757 08.66, +2709 46.4 & & & 28 & S \\
\hline 3 & $09.21,+270706.9$ & & & 50 & \\
\hline 4 & $75712.09,+270323.8$ & & & 46 & \\
\hline 5 & $75718.01,+270751.1$ & 16.92 & 291 & 44 & \\
\hline 6 & , +2704 57.8 & 15.72 & 9913 & 45 & \\
\hline 7 & , +270008.5 & 15.93 & 19633 & 43 & \\
\hline $8^{*}$ & $75724.02,+265846.9$ & 18.94 & 720155 & 395 & S \\
\hline 9 & $9,+272321.9$ & 18.37 & 30870 & 40 & \\
\hline 10 & 7, +272001.3 & 17.22 & 37076 & 39 & S \\
\hline 11 &,+270500.3 & 16.58 & 29492 & 41 & S \\
\hline 12 &,+271403.1 & 17.36 & 20638 & 23 & $\mathrm{~S}$ \\
\hline 13 & +271751.3 & 16.40 & 37 & 44 & $\mathrm{~S}$ \\
\hline 14 & , +27 2229.0 & 18.42 & & 43 & $\mathrm{~S}$ \\
\hline 15 & , +265042.6 & & 8076 & 16 & $\mathrm{~S}$ \\
\hline 16 & 757 & 17.70 & & 18 & $S$ \\
\hline 17 & 7574 & 17.52 & & 46 & $S$ \\
\hline 18 & 271 & & & 46 & $\mathrm{~S}$ \\
\hline 19 & 7572 & 19.09 & & 4 & $\mathrm{~S}$ \\
\hline 20 & 7574 & 17.38 & & 46 & $\mathrm{~S}$ \\
\hline 21 & 270145.5 & 16.95 & 1660 & 15 & $\sqrt{3}$ \\
\hline $22 *$ & 265514.7 & 18.69 & 89658 & 435 & $\mathrm{~S}$ \\
\hline 23 & +270722.9 & 17.60 & 1562 & 36 & $\mathrm{~W}+\mathrm{S}$ \\
\hline 24 & +270941.8 & 17.71 & 3929 & 12 & $W+S$ \\
\hline 25 & +272933.5 & 17.23 & & 49 & S \\
\hline 26 & +265931.1 & 17.40 & & 40 & $\mathrm{~W}+\mathrm{S}$ \\
\hline 27 & 7575 & 17.05 & & 19 & $w+S$ \\
\hline $28^{*}$ & 75758 & 19.00 & & 470 & S \\
\hline 29 & & & & 25 & $\mathrm{~S}$ \\
\hline 30 & & & & 37 & $\mathrm{~S}$ \\
\hline 31 & & & & 30 & $\mathrm{~W}+\mathrm{S}$ \\
\hline $32 *$ & 75 & & & 286 & $\mathrm{~S}$ \\
\hline 33 & 758 & 16.58 & & 30 & $\mathrm{~W}+\mathrm{S}$ \\
\hline 34 & 758 & 16. & 7769 & 30 & $\mathrm{~S}$ \\
\hline 35 & 7580 & 17.59 & 28924 & 33 & $\mathrm{~W}+\mathrm{S}$ \\
\hline 36 & 272104.7 & 16.57 & 6841 & 45 & $\mathrm{~S}$ \\
\hline 37 & 7581 & 17.46 & 287 & 31 & $W+S$ \\
\hline 38 & 7581 & 17. & & 14 & $\mathrm{~S}$ \\
\hline 39 & 3.9 & 18. & & 101 & W \\
\hline 40 & 9.6 & 14.51 & 138 & 39 & $W+S$ \\
\hline 41 & 581 & 14.68 & 386 & 24 & $\mathrm{~W}+\mathrm{S}$ \\
\hline $42 *$ & & 19. & & 237 & S \\
\hline 43 & & & & 38 & $\mathrm{~S}$ \\
\hline 44 & & & & 17 & $W+S$ \\
\hline 45 & & & & 39 & S \\
\hline 46 & & & & 42 & $S$ \\
\hline 47 & & & & 52 & \\
\hline 48 & 75 & & & 33 & $\mathrm{~V}$ \\
\hline 49 & 7583 & & & 33 & \\
\hline 50 & 27021 & 16. & & 29 & $W+S$ \\
\hline $51 *$ & , +27 1151.4 & 18. & & 261 & $\mathrm{~S}$ \\
\hline 52 & +27184 & 17. & & 6 & $W+S$ \\
\hline 53 & +271136.8 & 18. & & 74 & W \\
\hline 54 & +265147.7 & 17.77 & & 29 & S \\
\hline 55 & 584 & 16.79 & & 21 & $W+S$ \\
\hline 56 & 7584 & 17.12 & & 26 & $\mathrm{~W}+\mathrm{S}$ \\
\hline 57 & 7584 & 17.45 & & 21 & $\mathrm{~W}+\mathrm{S}$ \\
\hline 58 & $75846.68,+273451.9$ & 15.90 & 22936 & 51 & $\mathrm{~S}$ \\
\hline 59 & 75846 & & & 52 & $S$ \\
\hline 60 & 75847 & 16. & & 17 & $\mathrm{~S}$ \\
\hline 61 & $75847.96,+271706.0$ & 16.33 & 28705 & 31 & $\mathrm{~W}+\mathrm{S}$ \\
\hline
\end{tabular}

Table 1. continued.

\begin{tabular}{|c|c|c|c|c|c|}
\hline ID & $\alpha, \delta(\mathrm{J} 2000)$ & $r^{\prime}$ & ${ }_{(\mathrm{kn}}^{v}$ & $\begin{array}{c}\Delta v \\
\left.3^{-1}\right)\end{array}$ & Source \\
\hline 62 & $75849.67,+272755.9$ & 17.42 & 29039 & 35 & $\mathrm{~S}$ \\
\hline 63 & $75852.31,+272401.6$ & 18.23 & 20449 & 120 & W \\
\hline 64 & $75852.99,+271251.2$ & 17.62 & 29272 & 30 & $\mathrm{~W}+\mathrm{S}$ \\
\hline 65 & $75854.80,+271128.5$ & 17.63 & 28444 & 39 & $\mathrm{~S}$ \\
\hline 66 & $75856.31,+273324.7$ & 16.44 & 22937 & 56 & $\mathrm{~S}$ \\
\hline 67 & $75856.53,+270317.2$ & 17.39 & 13626 & 12 & $\mathrm{~S}$ \\
\hline 68 & $75858.20,+270937.1$ & 15.84 & 22629 & 31 & $\mathrm{~W}+\mathrm{S}$ \\
\hline 69 & $75859.33,+271256.8$ & 16.68 & 28846 & 39 & $\mathrm{~W}+\mathrm{S}$ \\
\hline 70 & $75900.21,+273533.9$ & 16.92 & 14101 & 29 & $\mathrm{~S}$ \\
\hline 71 & $75900.70,+273352.8$ & 17.01 & 37403 & 50 & $\mathrm{~S}$ \\
\hline 72 & $75901.04,+270712.1$ & 17.30 & 29007 & 29 & $\mathrm{~W}+\mathrm{S}$ \\
\hline 73 & $75901.10,+270943.3$ & 16.52 & 25836 & 26 & W \\
\hline 74 & $75901.28,+270611.8$ & 17.36 & 29190 & 35 & $\mathrm{~S}$ \\
\hline 75 & $75903.63,+265309.7$ & 17.05 & 22372 & 28 & $\mathrm{~W}+\mathrm{S}$ \\
\hline 76 & $75904.34,+270855.4$ & 16.86 & 28833 & 40 & $\mathrm{~S}$ \\
\hline 77 & $75904.34,+271325.6$ & 16.83 & 29178 & 38 & $\mathrm{~W}+\mathrm{S}$ \\
\hline 78 & $75905.16,+272734.3$ & 15.32 & 6741 & 54 & S \\
\hline 79 & $75905.81,+270629.8$ & 16.77 & 29277 & 35 & $\mathrm{~W}+\mathrm{S}$ \\
\hline 80 & $75906.22,+271539.4$ & 16.54 & 22300 & 78 & W \\
\hline 81 & $75906.80,+265054.4$ & 18.37 & 99637 & 46 & $\mathrm{~S}$ \\
\hline 82 & $75907.49,+271812.0$ & 17.41 & 24710 & 15 & $\mathrm{~W}+\mathrm{S}$ \\
\hline 83 & $75908.52,+271127.1$ & 17.41 & 29123 & 15 & $\mathrm{~W}+\mathrm{S}$ \\
\hline 84 & $75909.00,+273315.6$ & 17.44 & 23184 & 13 & $\mathrm{~S}$ \\
\hline 85 & $75910.27,+264907.1$ & 16.08 & 20469 & 44 & $\mathrm{~S}$ \\
\hline 86 & 759 11.37, +27 1050.7 & 17.31 & 30233 & 43 & W \\
\hline 87 & $75912.89,+270616.0$ & 16.96 & 30172 & 53 & W \\
\hline 88 & $75913.65,+270541.0$ & 16.14 & 29016 & 40 & $\mathrm{~W}$ \\
\hline 89 &,+263 & 17.56 & 6473 & 28 & $\mathrm{~S}$ \\
\hline 90 & $75916.02,+270857.7$ & 17.60 & 29242 & 23 & $\mathrm{~S}$ \\
\hline 91 & $75917.10,+270916.1$ & 15.19 & 29808 & 103 & W \\
\hline 92 & $7,+270440.3$ & 13.72 & 6808 & 50 & $\mathrm{~S}$ \\
\hline 93 & $75918.74,+270710.2$ & 17.42 & 27598 & 39 & $\mathrm{~S}$ \\
\hline 94 & $75920.05,+270950.4$ & 16.38 & 29693 & 36 & $\mathrm{~W}+\mathrm{S}$ \\
\hline 95 & $75924.05,+270849.5$ & 16.24 & 29901 & 38 & W \\
\hline 96 & $75924.24,+265732.4$ & 17.95 & 6299 & 56 & $\mathrm{~W}$ \\
\hline 97 & $75924.36,+271755.4$ & 17.52 & 22809 & 14 & $\mathrm{~W}+\mathrm{S}$ \\
\hline 98 & $75925.00,+270748.9$ & 15.72 & 27901 & 42 & $\mathrm{~W}+\mathrm{S}$ \\
\hline 99 & $75927.79,+270703.7$ & 17.07 & 28709 & 26 & $\mathrm{~W}+\mathrm{S}$ \\
\hline 100 & $75927.83,+270545.4$ & 15.97 & 20444 & 30 & $\mathrm{~W}+\mathrm{S}$ \\
\hline 101 & $75928.52,+265322.6$ & 14.24 & 7924 & 23 & $\mathrm{~S}$ \\
\hline 102 & , +270135.2 & 12.60 & 6654 & 59 & $\mathrm{~S}$ \\
\hline 103 & $75932.18,+263818.0$ & 15.87 & 22442 & 41 & $\mathrm{~S}$ \\
\hline 104 & , +265508.6 & 17.98 & 45163 & 79 & W \\
\hline 105 &,+264350.3 & 16.23 & 14062 & 16 & $\mathrm{~S}$ \\
\hline 106 & $75937.79,+265729.6$ & 17.43 & 29709 & 26 & $\mathrm{~W}+\mathrm{S}$ \\
\hline 107 & $75938.39,+273325.7$ & 17.17 & 24596 & 15 & $\mathrm{~S}$ \\
\hline 108 & $75940.16,+270514.1$ & 17.22 & 14234 & 20 & $\mathrm{~W}+\mathrm{S}$ \\
\hline 109 & $75940.18,+273030.9$ & 16.50 & 23160 & 41 & $\mathrm{~S}$ \\
\hline 110 & $75940.90,+273545.2$ & 16.54 & 22861 & 43 & $\mathrm{~S}$ \\
\hline 111 & $75941.72,+270012.6$ & 15.43 & 6642 & 27 & $\mathrm{~W}+\mathrm{S}$ \\
\hline 112 & $75942.16,+271917.0$ & 18.36 & 27799 & 134 & W \\
\hline 113 & $75943.49,+271010.9$ & 17.13 & 29631 & 43 & $\mathrm{~S}$ \\
\hline 114 & $75944.15,+272313.4$ & 16.94 & 47209 & 40 & $\mathrm{~S}$ \\
\hline 115 & $75946.49,+263819.2$ & 17.79 & 8121 & 17 & $\mathrm{~S}$ \\
\hline 116 & $75947.17,+273124.3$ & 17.58 & 31213 & 35 & $\mathrm{~S}$ \\
\hline 117 & $75948.38,+271137.1$ & 18.30 & 87323 & 34 & $\mathrm{~S}$ \\
\hline 118 & $75949.80,+270937.1$ & 17.90 & 20839 & 105 & W \\
\hline 119 & $75951.25,+265042.3$ & 17.12 & 49160 & 15 & $\mathrm{~W}+\mathrm{S}$ \\
\hline 120 & $75953.83,+264720.5$ & 15.12 & 14223 & 19 & $\mathrm{~S}$ \\
\hline 121 & $75954.14,+270123.5$ & 17.77 & 28725 & 34 & $\mathrm{~S}$ \\
\hline 122 & $75954.42,+270854.7$ & 17.59 & 30176 & 34 & $\mathrm{~S}$ \\
\hline 123 & $75955.14,+265035.2$ & 17.45 & 49281 & 60 & W \\
\hline 124 & $75955.22,+270747.8$ & 17.31 & 13978 & 13 & $\mathrm{~S}$ \\
\hline 125 & $75957.75,+265314.7$ & 17.57 & 29480 & 38 & $\mathrm{~S}$ \\
\hline 126 & $75959.13,+270902.5$ & 17.52 & 20722 & 84 & W \\
\hline
\end{tabular}


Table 1. continued.

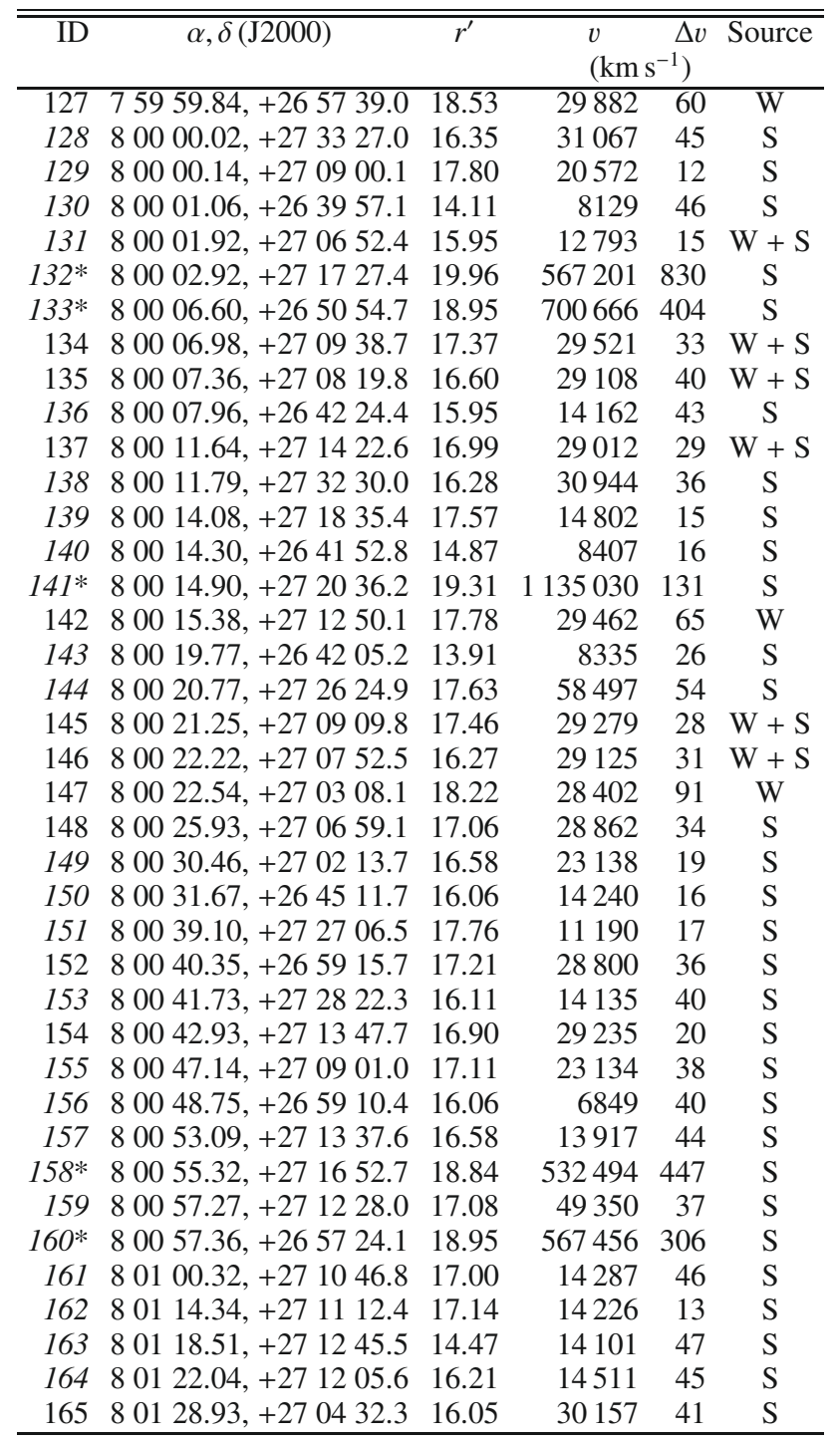

Table 4. Velocity catalog of 51 spectroscopically measured galaxies in the field of A725. In Col. 1, IDs in italics indicate non-cluster galaxies. ID 24 (in boldface) is BCMI (see text).

\begin{tabular}{|c|c|c|c|c|c|}
\hline ID & $\alpha, \delta(\mathrm{J} 2000)$ & $R$ & $B$ & \multicolumn{2}{|c|}{$\left(\mathrm{km} \mathrm{s}^{-1}\right)$} \\
\hline 1 & $085855.32,+624453.4$ & - & - & 8079 & 68 \\
\hline 2 & $085858.09,+623623.5$ & 16.18 & 18.35 & 26850 & 39 \\
\hline 3 & $085906.88,+623420.7$ & 17.' & & 27692 & 63 \\
\hline 4 & $085917.09,+623321.3$ & 16. & & & 52 \\
\hline 5 & $085932.75,+622045.9$ & 16.88 & 18.17 & 7389 & 68 \\
\hline 6 & $085936.97,+622046.3$ & - & - & 27473 & 82 \\
\hline 7 & $085941.35,+623916.4$ & 16.53 & 18.50 & 49663 & 95 \\
\hline 8 & $085947.36,+623758.6$ & 17.68 & 19.14 & 28261 & 70 \\
\hline 9 & $085955.76,+623555.6$ & 17.45 & 19.27 & 28561 & 90 \\
\hline 10 & $090022.25,+624329.2$ & 16.61 & 18.61 & 26269 & 47 \\
\hline 11 & $090022.42,+621907.5$ & - & - & 13495 & 49 \\
\hline 12 & $090025.98,+623745.8$ & 16.52 & 18.69 & 27461 & 49 \\
\hline 13 & $090032.55,+624200.8$ & 16.81 & 18.90 & 27293 & 60 \\
\hline 14 & $090035.62,+624359.4$ & 16.83 & 18.51 & 27457 & 79 \\
\hline 15 & $090037.96,+625033.7$ & 17.37 & 19.18 & 50005 & 37 \\
\hline 16 & $090038.09,+623805.7$ & 16.67 & 18.66 & 27620 & 105 \\
\hline 17 & $090042.36,+623449.2$ & 16.86 & 19.04 & 27414 & 117 \\
\hline 18 & $090048.50,+62355$ & 16.79 & 18.29 & 471 & 53 \\
\hline 19 & $8,+623749.5$ & 15.57 & 17.64 & 29360 & 48 \\
\hline 20 & $090055.72,+623006.6$ & 18.25 & 19.84 & 53737 & 52 \\
\hline 21 & $090057.04,+623257.8$ & 15.91 & 18.12 & 27235 & 24 \\
\hline 22 & $090058.85,+623831.9$ & 16.09 & 16.87 & 27739 & 49 \\
\hline 23 & $090103.65,+623652.9$ & 15.62 & 17.67 & 28023 & 45 \\
\hline 24 & $090109.99,+623720.0$ & 15.12 & 16.41 & 27104 & 48 \\
\hline 25 & $090111.08,+623927.9$ & 16.61 & 18.70 & 28324 & 42 \\
\hline 26 & $090114.79,+623610.6$ & 15.44 & 17.59 & 27449 & 22 \\
\hline 27 & $090134.70,+623716.1$ & 16.99 & 19.14 & 27616 & 103 \\
\hline 28 & $090134.95,+623332.7$ & 17.56 & 18.63 & 25973 & 23 \\
\hline 29 & $090135.40,+623321.1$ & 17.75 & 19.12 & 30626 & 147 \\
\hline 30 &,+623 & 15.79 & 17.80 & 27716 & 43 \\
\hline 31 & 140.93, +62 3759.9 & 17.01 & 19.05 & 27942 & 83 \\
\hline 32 & $090149.97,+624233.6$ & 16. & 18 & 270 & 60 \\
\hline 33 & +623 & 18.15 & 19.20 & 27537 & 155 \\
\hline 34 & $090152.15,+623412.3$ & 16.08 & 18.25 & 26873 & 75 \\
\hline 35 & $090206.50,+625440.7$ & - & - & 90943 & 101 \\
\hline 36 & $090207.78,+624040.7$ & 16.27 & 18.24 & 26846 & 84 \\
\hline 37 & $090209.60,+621930.4$ & - & - & 11134 & 122 \\
\hline 38 & $090209.98,+624501.1$ & 17.70 & 19.65 & 27530 & 75 \\
\hline 39 & $090215.21,+624016.4$ & 14.88 & 17.16 & 27108 & 48 \\
\hline 40 & $090216.28,+623225.7$ & 17.66 & 19.65 & 53311 & 57 \\
\hline 41 & $090219.33,+623858.1$ & 17.40 & 19.18 & 27659 & 68 \\
\hline 42 & $090219.51,+624814.1$ & 17.66 & 19.16 & 28592 & 95 \\
\hline 43 & $090219.58,+622216.3$ & 17.46 & 19.74 & 48789 & 91 \\
\hline 44 & $090220.67,+624544.8$ & 17.72 & 19.58 & 52418 & 68 \\
\hline 45 & $090229.76,+622831.3$ & 16.77 & 18.75 & 26878 & 130 \\
\hline 46 & $57.75,+622200.1$ & - & - & 62471 & 71 \\
\hline 47 & $090258.11,+624845.0$ & - & - & 27632 & 79 \\
\hline 48 & $090258.30,+623856.7$ & - & - & 22394 & 47 \\
\hline 49 & $090301.38,+623931.4$ & - & - & 22240 & 103 \\
\hline 50 & 090306.77, +62 4939.6 & - & - & 27267 & 73 \\
\hline 51 & $090313.73,+623832.5$ & - & - & 50197 & 83 \\
\hline
\end{tabular}


W. Boschin et al.: Optical analysis of poor radio clusters, Online Material $p 3$

Table 5. Velocity catalog of 99 spectroscopically measured galaxies in the field of A796. In Col. 1, IDs in italics indicate non-cluster galaxies. IDs 54 and 56 (in boldface) are the brightest cluster members. Asterisks in Col. 1 highlight QSOs in the SDSS catalog.

\begin{tabular}{|c|c|c|c|c|c|}
\hline ID & $\begin{array}{c}\alpha, \delta(\mathrm{J} 2000) \\
09^{\mathrm{h}},+60^{\circ}\end{array}$ & \multicolumn{4}{|c|}{$\left(\mathrm{km} \mathrm{s}^{-1}\right)$} \\
\hline $1^{*}$ & $2403.66,2243.1$ & 18.39 & 289995 & 375 & $\mathrm{~S}$ \\
\hline $2 *$ & $2424.87,1459.2$ & 18.61 & 644626 & 405 & $\mathrm{~S}$ \\
\hline 3 & $2444.13,1353.3$ & 17.66 & 68395 & 54 & $\mathrm{~S}$ \\
\hline 4 & $2445.38,1555.2$ & 17.52 & 59869 & 54 & S \\
\hline 5 & $2507.51,0452.1$ & 19.27 & 134817 & 84 & $\mathrm{~S}$ \\
\hline $6^{*}$ & $2513.33,1039.2$ & 17.68 & 254929 & 498 & $\mathrm{~S}$ \\
\hline 7 & $2526.14,3141.4$ & 17.49 & 77745 & 57 & $\mathrm{~S}$ \\
\hline 8 & $2536.67,2235.7$ & 17.38 & 79529 & 48 & $\mathrm{~S}$ \\
\hline 9 & $2539.80,0729.9$ & 17.23 & 47241 & 51 & $\mathrm{~S}$ \\
\hline 10 & $2550.79,1713.2$ & 19.38 & 47187 & 78 & W \\
\hline $11 *$ & 25 59.93, 2648.5 & 18.17 & 191738 & 492 & $\mathrm{~S}$ \\
\hline 12 & $2603.83,3602.6$ & 17.19 & 46654 & 45 & $\mathrm{~S}$ \\
\hline $13 *$ & $2612.49,2102.7$ & 19.27 & 553642 & 525 & $\mathrm{~S}$ \\
\hline $14^{*}$ & $2614.75,2558.4$ & 19.39 & 670723 & 462 & $\mathrm{~S}$ \\
\hline 15 & $2619.73,3015.7$ & 18.12 & 77784 & 45 & $\mathrm{~S}$ \\
\hline 16 & $2620.66,3301.5$ & 16.91 & 33780 & 38 & $\mathrm{~W}+\mathrm{S}$ \\
\hline 17 & $2623.36,4220.1$ & 17.32 & 30615 & 18 & S \\
\hline 18 & $2625.94,0509.6$ & 17.63 & 46216 & 30 & $\mathrm{~S}$ \\
\hline 19 & $2630.12,4830.3$ & 17.00 & 43218 & 42 & $\mathrm{~S}$ \\
\hline 20 & $2630.33,2852.9$ & 17.96 & 90115 & 45 & $\mathrm{~S}$ \\
\hline 21 & $2634.66,2339.8$ & 17.93 & 39946 & 57 & W \\
\hline 22 & $2637.08,2339.4$ & 17.73 & 39701 & 45 & $\mathrm{~S}$ \\
\hline 23 & $2639.52,3701.0$ & 16.97 & 21478 & 70 & $\mathrm{~W}+\mathrm{S}$ \\
\hline 24 & $2644.25,0650.7$ & 17.69 & 75955 & 42 & S \\
\hline 25 & $2646.11,0339.7$ & 17.21 & 47418 & 48 & $\mathrm{~S}$ \\
\hline 26 & $2646.15,3128.8$ & 18.41 & 90825 & 42 & $\mathrm{~S}$ \\
\hline 27 & $2649.47,2724.2$ & 18.22 & 45262 & 56 & W \\
\hline 28 & $2649.88,0217.2$ & 17.70 & 47499 & 45 & $\mathrm{~S}$ \\
\hline 29 & $2650.12,2436.7$ & 17.60 & 39648 & 42 & $\mathrm{~S}$ \\
\hline 30 & $2651.06,1204.3$ & 19.00 & 10952 & 139 & W \\
\hline $31 *$ & $2653.67,2217.4$ & 20.17 & 455885 & 468 & $\mathrm{~S}$ \\
\hline 32 & $2653.98,1927.0$ & 17.91 & 47066 & 69 & W \\
\hline 33 & $2655.38,0649.3$ & 18.91 & 53621 & 64 & W \\
\hline $34^{*}$ & $2656.95,2504.3$ & 19.14 & 886148 & 13317 & $\mathrm{~S}$ \\
\hline 35 & $2659.88,4805.4$ & 15.39 & 30681 & 45 & $\mathrm{~S}$ \\
\hline 36 & $2701.69,0337.3$ & 17.67 & 53360 & 45 & $\mathrm{~S}$ \\
\hline 37 & $2707.68,3150.7$ & 17.61 & 46872 & 75 & W \\
\hline 38 & $2707.80,3227.3$ & 17.26 & 43198 & 37 & $\mathrm{~W}+\mathrm{S}$ \\
\hline 39 & $2714.41,3843.9$ & 19.02 & 54241 & 58 & W \\
\hline 40 & 27 14.95, 2249.6 & 18.48 & 30743 & 48 & W \\
\hline 41 & $2716.64,3502.6$ & 17.57 & 43341 & 33 & $\mathrm{~W}+\mathrm{S}$ \\
\hline 42 & $2725.60,2216.2$ & 16.46 & 30522 & 29 & $\mathrm{~W}+\mathrm{S}$ \\
\hline 43 & $2729.10,1951.0$ & 17.80 & 69182 & 31 & W \\
\hline 44 & 27 29.38, 2707.8 & 17.72 & 46717 & 34 & W \\
\hline 45 & $2734.31,1107.5$ & 17.05 & 22480 & 40 & $\mathrm{~W}+\mathrm{S}$ \\
\hline 46 & $2740.19,2747.2$ & 17.58 & 46767 & 31 & $\mathrm{~W}+\mathrm{S}$ \\
\hline 47 & $2740.83,2650.4$ & 18.16 & 45407 & 110 & W \\
\hline 48 & $2742.87,4024.9$ & 18.65 & 33263 & 109 & W \\
\hline 49 & $2744.28,0839.3$ & 18.29 & 21315 & 98 & W \\
\hline 50 & $2747.09,3100.9$ & 17.87 & 46921 & 43 & W \\
\hline 51 & $2752.10,3401.7$ & 17.73 & 39922 & 83 & W \\
\hline 52 & $2753.74,2420.3$ & 16.52 & 1286 & 39 & $\mathrm{~S}$ \\
\hline 53 & 27 58.61, 2617.7 & 17.29 & 46961 & 122 & W \\
\hline 54 & $2800.65,2626.8$ & 16.96 & 46038 & 29 & $\mathrm{~W}+\mathrm{S}$ \\
\hline 55 & $2803.20,2415.7$ & 18.72 & 47229 & 43 & W \\
\hline 56 & $2810.75,2815.0$ & 16.94 & 46934 & 39 & $\mathrm{~W}+\mathrm{S}$ \\
\hline 57 & $2811.89,4519.8$ & 16.94 & 39600 & 42 & S \\
\hline 58 & $2812.80,2216.7$ & 17.62 & 47534 & 37 & $\mathrm{~W}+\mathrm{S}$ \\
\hline 59 & $2814.76,0207.5$ & 17.59 & 30048 & 39 & S \\
\hline 60 & $2819.76,4947.3$ & 16.26 & 26987 & 48 & $\mathrm{~S}$ \\
\hline 61 & $2824.54,1419.4$ & 16.97 & 22797 & 20 & $\mathrm{~W}+\mathrm{S}$ \\
\hline 62 & $2824.54,2334.8$ & 17.84 & 47290 & 40 & W \\
\hline
\end{tabular}

Table 5. continued.

\begin{tabular}{|c|c|c|c|c|c|}
\hline ID & $\begin{array}{c}\alpha, \delta(\mathrm{J} 2000) \\
09^{\mathrm{h}},+60^{\circ}\end{array}$ & $r^{\prime}$ & ${ }^{v}(\mathrm{~km}$ & ${ }_{-1} \Delta v$ & Source \\
\hline 63 & $2827.29,2724.1$ & 18.77 & 46554 & 58 & $\mathrm{~W}$ \\
\hline 64 & $2833.51,1845.6$ & 18.79 & 35964 & 66 & W \\
\hline $65^{*}$ & $2837.98,2521.0$ & 16.86 & 88574 & 477 & $\mathrm{~S}$ \\
\hline 66 & $2841.44,0516.6$ & 18.40 & 48770 & 64 & W \\
\hline 67 & $2844.90,2545.8$ & 17.40 & 46204 & 39 & $\mathrm{~S}$ \\
\hline 68 & $2850.43,4840.9$ & 18.30 & 88076 & 48 & $\mathrm{~S}$ \\
\hline 69 & $2855.52,5133.6$ & 17.56 & 73272 & 45 & $\mathrm{~S}$ \\
\hline 70 & $2857.65,5249.4$ & 17.09 & 35864 & 45 & $\mathrm{~S}$ \\
\hline 71 & $2859.48,2050.4$ & 16.96 & 22734 & 37 & $\mathrm{~W}+\mathrm{S}$ \\
\hline 72 & $2915.19,1456.9$ & 19.36 & 47819 & 62 & W \\
\hline $73^{*}$ & $2918.14,1706.3$ & 19.03 & 665093 & 435 & $\mathrm{~S}$ \\
\hline 74 & $2927.43,1803.4$ & 17.16 & 35941 & 35 & $W+S$ \\
\hline 75 & $2929.47,0251.2$ & 17.38 & 48884 & 42 & S \\
\hline 76 & $2932.16,3709.1$ & 17.13 & 35963 & 38 & $W+S$ \\
\hline 77 & $2935.13,3306.2$ & 17.30 & 44039 & 41 & $W+S$ \\
\hline 78 & $2950.93,1454.6$ & 16.92 & 47715 & 45 & S \\
\hline 79 & $2955.21,2317.2$ & 16.25 & 36079 & 23 & $W+S$ \\
\hline 80 & $2958.50,1204.6$ & 18.39 & 80522 & 62 & W \\
\hline 81 & $2959.36,1446.1$ & 17.62 & 54170 & 39 & $\mathrm{~W}+\mathrm{S}$ \\
\hline $82 *$ & $3004.28,3930.0$ & 18.43 & 233694 & 345 & $\mathrm{~S}$ \\
\hline 83 & $3006.44,2653.4$ & 16.93 & 4086 & 3 & $\mathrm{~S}$ \\
\hline 84 & 30 09.12, 2805.6 & 16.29 & 4072 & 21 & $W+S$ \\
\hline 85 & $3014.03,2104.6$ & 17.66 & 42978 & 32 & $\mathrm{~W}+\mathrm{S}$ \\
\hline 86 & $3026.63,2648.8$ & 18.72 & 11766 & 100 & W \\
\hline 87 & $3030.62,2205.0$ & 18.34 & 50023 & 35 & W \\
\hline 88 & $3033.74,1217.6$ & 17.56 & 46159 & 42 & S \\
\hline 89 & $3045.28,1423.0$ & 17.51 & 65622 & 51 & $\mathrm{~S}$ \\
\hline $90^{*}$ & 30 51.94, 2301.1 & 19.49 & 1111330 & 240 & $\mathrm{~S}$ \\
\hline 91 & $3055.40,0436.7$ & 19.30 & 163600 & 69 & $\mathrm{~S}$ \\
\hline 92 & $3105.10,1619.5$ & 17.14 & 42900 & 42 & $\mathrm{~S}$ \\
\hline 93 & $3105.32,1401.1$ & 17.30 & 53867 & 45 & $\mathrm{~S}$ \\
\hline 94 & $3109.48,1459.9$ & 15.69 & 7765 & 27 & $\mathrm{~S}$ \\
\hline 95 & $3111.54,3744.5$ & 17.53 & 33835 & 33 & $\mathrm{~S}$ \\
\hline 96 & $3113.88,0629.1$ & 19.12 & 187991 & 417 & $\mathrm{~S}$ \\
\hline 97 & $3117.95,3603.2$ & 16.46 & 44219 & 45 & $\mathrm{~S}$ \\
\hline 98 & $3125.83,1650.4$ & 17.50 & 43062 & 42 & $\mathrm{~S}$ \\
\hline $99 *$ & $3130.71,1106.3$ & 19.00 & 436147 & 687 & $\mathrm{~S}$ \\
\hline
\end{tabular}

